\title{
Circulant Synthesis of Central Pattern Generators With Application to Control of Rectifier Systems
}

\author{
Zhiyong Chen, Member, IEEE, and Tetsuya Iwasaki, Senior Member, IEEE
}

\begin{abstract}
While regulations around an equilibrium point or a reference trajectory have been the focus of recent feedback control theories, generation of autonomous oscillations with a specific pattern plays a crucial role in important control applications such as robotics. The central pattern generator (CPG) is the fundamental neuronal mechanism underlying rhythmic movements of animals, and may provide a new paradigm for controlled oscillations of engineering systems. This paper gives a novel method for synthesizing artificial CPG circuits by utilizing the properties of circulant matrices. We show how neuron models can be interconnected to yield a periodic orbit with prescribed frequency, amplitudes, and phases. Using the synthetic CPG in a feedback loop, we propose a control design method for a class of nonlinear rectifier systems to achieve the optimal state pattern with respect to a certain measure of efficiency, subject to small ripples in the rectified variable. The effectiveness of the proposed method is illustrated by an application to the gait control of an undulatory snakelike system.
\end{abstract}

Index Terms-Animal locomotion, central pattern generator (CPG), neuronal control, nonlinear oscillator.

\section{INTRODUCTION}

A NIMAL LOCOMOTIONS such as walking, swimming, flying, and crawling are realized by coordinated body oscillations interacting with the surrounding environment. For example, the undulatory body motion of a snake is transformed into its forward velocity through frictional contacts with the ground. Rhythmic body movements in animal locomotion are known to be controlled by certain neuronal elements called the central pattern generator (CPG). Neuroscientists have modeled the CPGs as nonlinear oscillators for various vertebrates and invertebrates, including crione, leech, lamprey, and crayfish [1]-[5]. Such models are developed so that the oscillations of cell membrane potentials observed in an isolated nerve cord are accurately reproduced as a stable limit cycle. A current focus of this line of research is on how the sensory feedback to the CPG modifies oscillation properties [6]-[8].

The biological control mechanism has motivated the use of CPG circuits as the basic architectures of nonlinear con-

Manuscript received March 23, 2006; revised May 1, 2007. This work was supported in part by the National Science Foundation (NSF) CAREER Award 0237708 and in part by the NIH/NINDS 1 R01 NS46057-01 as part of the NSF/NIH Collaborative Research in Computational Neuroscience Program. Recommended by Associate Editor D. Angeli.

Z. Chen is with the School of Electrical Engineering and Computer Science, The University of Newcastle, Callaghan, N.S.W. 2308, Australia (e-mail: zhiyong.chen@newcastle.edu.au).

T. Iwasaki is with the Department of Mechanical and Aerospace Engineering, University of Virginia, Charlottesville, VA 22904-4746 USA (e-mail: iwasaki@virginia.edu).

Color versions of one or more of the figures in this paper are available online at http://ieeexplore.ieee.org.

Digital Object Identifier 10.1109/TAC.2007.914951 trol laws to achieve coordinated oscillations of engineered systems [9]-[12]. Such systems are typically realized by feedback connections of nonlinear oscillators and mechanical dynamics. A fundamental problem in locomotion control is to regulate some of the closed-loop states (e.g., global velocity) to a set point through autonomous, coordinated oscillations of other states (e.g., relative angles of body parts). This is in contrast with the traditional control problems where regulation or tracking is essentially achieved via stabilization around an equilibrium. In view of robustness and autonomy of biological control mechanisms, CPGs may provide a new paradigm for nonlinear feedback control theories to complement the existing approaches [13], [14] that achieve coordinated oscillations. However, the designs of CPG-based controllers so far have relied largely on heuristic tuning of parameters, guided by some biological principles. At the moment, there is no systematic method for designing a feedback controller within the CPG framework to achieve a prescribed oscillation pattern for the closed-loop states.

In the first part of the paper, we consider the problem of designing CPGs to achieve desired oscillation patterns without sensory feedback. A typical behavior of a CPG consisting of $m$ neurons may be represented by phase-locked oscillations of the membrane potentials $z_{i}(t)(i=1, \ldots, m)$ with similar wave forms. Hence, the oscillation pattern of CPGs is essentially characterized by $z_{i}(t)=a_{i} \mathcal{Q}\left(\varpi t+\phi_{i}\right)$ in terms of the frequency $\varpi$, amplitudes $a_{i}$, phases $\phi_{i}$, and wave form $\mathcal{Q}$ that is a $2 \pi$-periodic function with unit amplitude, i.e., $\max |\mathcal{Q}(t)|=1$. Our main result will show how an oscillator of a generalized CPG structure can be designed so that every nontrivial trajectory converges to a periodic orbit specified by $\varpi, a_{i}$, and $\phi_{i}$. The wave form $\mathcal{Q}$ is unspecified a priori in the design, but is shown to approach a sinusoid as the number of scalar nonlinearities in the oscillator becomes larger. The key idea behind our development is the circulant structure of neuronal interconnections, leading to a uniform phase spacing. By projecting the states onto an appropriate subspace, any desired phases can be achieved exactly, provided $\phi_{i} /(2 \pi)$ are rational.

There are results in the literature that address oscillator analysis/design problems [15]-[19]. We note, in particular, that partial contraction analysis was used in [18] to prove global exponential convergence to a desired phase-locked state. However, the class of CPG models considered in these references are different from ours. Specifically, the previous results showed how a given set of oscillators should be coupled to achieve desired phase relations among the oscillators. On the other hand, our result shows how a given set of nonoscillatory subsystems (nonspiking neuron models) can be interconnected to achieve periodic 
motions with desired phase relations among the subsystems. The circulant oscillators proposed in this paper may be used as a basic unit in further study of coupled oscillators. Finally, it is worth noting that the circulant interconnection architecture has recently been exploited in the context of cooperative control of multiagent systems [20].

In the second part of this paper, we discuss an application of the circulant CPGs to the control of dynamical rectifier systems. We call a system a dynamical rectifier if certain coordinated oscillations of the inputs result in biased outputs. Such rectifier systems represent essential dynamics associated with animal locomotion where rhythmic body movements are rectified to generate forward velocity. We consider a modified version of Brockett's cyclic systems [14], where the bilinear mechanism $w:=z^{\mathrm{T}} \Lambda \dot{z}$ captures the effect of rectification. If the matrix $\Lambda$ is not symmetric, then periodic variation of $z(t)$ renders the average value of $w(t)$ over a cycle nonzero. Thus, oscillations of $z(t)$ are rectified to yield nonzero bias in $w(t)$. We propose a method for designing feedback controllers to generate stable limit cycles in the states $z(t)$ that lead to a desired value of the rectified signal $w(t)$ on average. The idea is to choose the feedback law so that the closed-loop system becomes a circulant oscillator. We also show how the "ripples" in $w(t)$ [i.e., periodic fluctuation of $w(t)$ from its average value] can be made arbitrarily small by making $z(t)$ oscillate in a quasi-sinusoidal manner with a specific phase property determined from $\Lambda$.

Notation: The set of real circulant matrices is defined by

$$
\begin{aligned}
\mathbb{O} & :=\left\{O(x) \in \mathbb{R}^{n \times n} \mid x \in \mathbb{R}^{n}\right\} \\
O(x) & :=\left[\begin{array}{cccc}
x_{n} & x_{1} & \cdots & x_{n-1} \\
x_{n-1} & x_{n} & \ddots & \vdots \\
\vdots & \ddots & \ddots & x_{1} \\
x_{1} & \cdots & x_{n-1} & x_{n}
\end{array}\right] .
\end{aligned}
$$

The matrix $J:=O(1,0, \ldots, 0)$ is called the basic circulant permutation matrix. Some properties of circulant matrices can be found for example in [21]-[23]. Let the set of integers be denoted by $\mathbb{Z}$, the set of positive integers up to $n$ by $\mathbb{Z}_{n}$, and define its subset $\mathbb{Z}_{n}^{-}$by

$$
k \in \mathbb{Z}_{n}^{-} \Leftrightarrow k \in\{1,2, \ldots, n-1\}, \quad k \neq \frac{n}{2} .
$$

Note that $\mathbb{Z}_{n}^{-}$is nonempty if and only if $n \geq 3$. For a matrix $M$, its transpose, Moore-Penrose inverse, and complex conjugate are denoted by $M^{\mathrm{T}}, M^{\dagger}$, and $\bar{M}$, respectively, and let $M^{*}:=$ $\bar{M}^{T}$. The real and imaginary parts are denoted by $\Re(\cdot)$ and $\Im(\cdot)$, respectively. For a family of functions $x(t)$ parametrized by $\epsilon$, we write $x(t)=O(\epsilon)$ to mean that there exists $c \in \mathbb{R}$, independent of $\epsilon$, such that $|x(t)| \leq c \epsilon$ holds for all $t$.

\section{CPG DESIGN FRAMEWORK}

\section{A. Problem Formulation}

The neuronal CPG underlying rhythmic animal movements may be modeled, in its simplest form, by

$$
x+\tau \dot{x}=M \varphi(x)
$$

where $x(t) \in \mathbb{R}^{n}, M \in \mathbb{R}^{n \times n}, \varphi: \mathbb{R}^{n} \rightarrow \mathbb{R}^{n}$ is a nonlinear function, and $\tau \in \mathbb{R}$ is a positive scalar. The structure in (1) arises from interconnections of $n$ neurons modeled by a firstorder low-pass filter and a static nonlinearity. The former captures the time lag associated with the synaptic interaction and the cell membrane dynamics, while the latter captures the threshold and saturation effects. The matrix $M$ specifies how the $n$ neuron models are interconnected, and will be referred to as the connectivity matrix. In engineering applications, we may add more flexibility in oscillators to enrich the signals they can produce, e.g., we may consider a generalized model

$$
z+\tau \dot{z}=M \varphi(L z)
$$

where $z(t) \in \mathbb{R}^{m}, M \in \mathbb{R}^{m \times n}, L \in \mathbb{R}^{n \times m}$, and $\varphi: \mathbb{R}^{n} \rightarrow \mathbb{R}^{n}$. Clearly, (2) reduces exactly to (1) if $m=n$ and $L=I$. We shall address the following problem.

CPG synthesis problem: Given $\varpi \in \mathbb{R}, a \in \mathbb{R}^{m}$, and $\phi \in \mathbb{R}^{m}$, find the parameters $\tau, M, L$, and nonlinearity $\varphi$, such that every nontrivial trajectory of the system (2) converges to a limit cycle given by

$$
z_{i}(t)=a_{i} \mathcal{Q}\left(\varpi t+\phi_{i}\right), \quad i \in \mathbb{Z}_{m}
$$

for some $2 \pi$-periodic signal $\mathcal{Q}(t) \in \mathbb{R}$ with unit amplitude, i.e., $\max |\mathcal{Q}(t)|=1$.

In the problem statement, $m$ is a given integer, while $n$ is a design parameter to be found. For technical reasons, we make the following assumption on the desired phases.

Assumption 1:

1) $\phi_{i} /(2 \pi)$ with $i \in \mathbb{Z}_{m}$ are distinct rational numbers.

2) There exist $i, j \in \mathbb{Z}_{m}$ such that $\phi_{i}-\phi_{j} \neq \ell \pi$ for any integer $\ell$.

Remark 2.1: This assumption imposes essentially no restrictions on the phases to be achieved because any desired phases can be approximated by those satisfying the assumption with an arbitrary accuracy. Note that $\left|\phi_{i}\right|$ can be greater than $2 \pi$ and this allows for the phase specification to include synchronization of states $z_{i}$ and $z_{j}$ by choosing, e.g., $\phi_{i}=\phi_{j}+2 \pi$. Thus, the distinctness assumption on $\phi_{i}$ can be imposed without loss of generality, and will turn out to be convenient for our development. For the CPG design, the phase specification will be given in terms of integers $n, k$, and $\eta_{i}$ as follows

$$
\phi_{i}=\eta_{i} \theta_{k}, \quad \theta_{k}:=\frac{2 \pi k}{n}, \quad k \in \mathbb{Z}_{n}^{-}, \quad \eta_{i} \in \mathbb{Z}_{n}
$$

for each $i \in \mathbb{Z}_{m}$. If there are synchronization requirements as described earlier, then the parameter $k$ would be chosen to be equal to the number of states to be synchronized. If there is no such requirement, then $k$ can be chosen as $k=1$. Note that $k=n$ and $k=n / 2$ are excluded by $k \in \mathbb{Z}_{n}^{-}$to avoid violation of part 2) of Assumption 1. This, in turn, necessitates $n \geq 3$ because otherwise $\mathbb{Z}_{n}^{-}$is empty. For a given set of phases $\phi_{i}$, its "realization" $\left(n, k, \eta_{i}\right)$ is not unique. For practical purposes, the minimal realization with the smallest $n$ may be preferred for simplicity since this $n$ turns out to be the input/output dimension of the nonlinearity $\varphi$ in (2). 
In our development that follows, we show that a solution to the CPG synthesis problem can be given using the following class of nonlinear functions.

Assumption 2: The function $\varphi$ is of the form $\varphi=\operatorname{diag}$ $(\psi, \ldots, \psi)$ where $\psi: \mathbb{R} \rightarrow \mathbb{R}$ is a locally Lipschitz, differentiable, scalar nonlinearity satisfying the following conditions for all nonzero $x \in \mathbb{R}: 1)|\psi(x)| \leq 1$; 2) $\psi(0)=0$; 3) $\psi^{\prime}(x) \geq 0$; 4) $\psi^{\prime}(0)=1$; 5) $|\psi(x)| \leq|x|$; and 6) $\psi^{\prime}(x)<\psi(x) / x$.

Remark 2.2: Part 1) means that the function is bounded, and the upper bound has been normalized to be one. Parts 2)-4) imply that the function is strictly in the first and third quadrants. Parts 5) and 6) imply $\left|\psi^{\prime}(x)\right| \leq 1$ for all $x \neq 0$. Thus, the slope of $\psi$ is maximum at $x=0$ with $\psi^{\prime}(0)=1$. Part 6$)$ is satisfied if $\psi^{\prime}(x)$ is monotonically decreasing in $x \in(0, \infty)$ and monotonically increasing in $x \in(-\infty, 0)$. If $\psi^{\prime \prime}(x)$ exists, then this sufficient condition reduces to $x \psi^{\prime \prime}(x)<0$ for all nonzero $x \in \mathbb{R}$. A typical example of the function $\psi(x)$ satisfying Assumption 2 is $\tanh (x)$.

\section{B. Outline of the Circulant Approach}

In this section, we will outline our approach to the CPG synthesis problem. We first consider the design of uniform phase oscillators (UPOs) where the phases are equally spaced over $2 \pi k$, i.e., $\phi_{i}$ are given by (4) with $\eta_{i}:=i$ and $m=n$. For simplicity, we remove the specifications of the frequency and amplitudes and set our initial design goal to achieve the uniform phases only.

The multivariable harmonic balance analysis [24] predicts that a solution to the UPO synthesis problem may be given by an CPG with a circulant connectivity structure. To prove that it is indeed a solution, we will show that the circulant CPG is equivalent, in the steady state, to a planar (two-dimensional) oscillator. Thus, the UPO design problem boils down to the analysis of a basic planar oscillator with an embedded circulant structure. This fact will be the conclusion of this section, and will be the basis for the developments in the sections that follow.

To this end, let us consider the system (1) with $\tau=1$. If $x_{i}$ is approximately sinusoidal with frequency $\omega_{o}$, amplitude $\alpha_{o}$, and uniform phase $\phi_{i}:=i \theta_{k}$, that is, $x_{i}(t) \cong \alpha_{o} \sin \left(\omega_{o} t+\phi_{i}\right)$, then the following harmonic balance equation holds approximately (see [24])

$$
M \chi=\lambda \chi, \quad \chi_{i}:=\alpha_{o} e^{j \phi_{i}}, \quad \lambda:=\frac{\left(1+j \omega_{o}\right)}{\kappa\left(\alpha_{o}\right)}
$$

where $\kappa$ is the describing function of $\psi$. Hence, the connectivity matrix $M$ to achieve the given oscillation profile can be estimated by solving the aforementioned equation. A real solution is obtained by choosing the eigenvalues at $\lambda, \bar{\lambda}$, and zero, as follows:

$$
M=\left[\begin{array}{ll}
\chi & \bar{\chi}
\end{array}\right]\left[\begin{array}{ll}
\lambda & 0 \\
0 & \bar{\lambda}
\end{array}\right]\left[\begin{array}{ll}
\chi & \bar{\chi}
\end{array}\right]^{\dagger}=R \Omega R^{\mathrm{T}}
$$

where

$$
R:=\left[\begin{array}{ll}
s_{k} & c_{k}
\end{array}\right], \quad \Omega:=\frac{2}{n \hbar_{o}}\left[\begin{array}{cc}
1 & -\omega_{o} \\
\omega_{o} & 1
\end{array}\right], \hbar_{o}:=\kappa\left(\alpha_{o}\right)
$$

$$
\begin{aligned}
c_{k} & :=\left[\begin{array}{llll}
\cos \left(\theta_{k}\right) & \cos \left(2 \theta_{k}\right) & \cdots & \cos \left(n \theta_{k}\right)
\end{array}\right]^{\mathrm{T}} \\
s_{k} & :=\left[\begin{array}{llll}
\sin \left(\theta_{k}\right) & \sin \left(2 \theta_{k}\right) & \cdots & \sin \left(n \theta_{k}\right)
\end{array}\right]^{\mathrm{T}} .
\end{aligned}
$$

Since the harmonic balance method is approximate in nature, the resulting oscillations are not guaranteed to have the desired frequency, amplitude, nor phases. In fact, even the existence of a periodic orbit is not guaranteed at this point. We shall investigate what definitive statements can be made on the system thus constructed, regarding the existence and properties of a periodic orbit. The key idea for this investigation is that the connectivity matrix $M$ in (5) is a circulant matrix. The proof of the following lemma is given in Appendix.

Lemma 2.1: Let $k, n \in \mathbb{Z}$ and $\omega_{o}, \hbar_{o} \in \mathbb{R}$ be given where $n$ and $\hbar_{o}$ are nonzero, and define $M$ by (5). Then, $M$ is a circulant matrix described by

$$
M=\frac{2}{n \hbar_{o}} O\left(c_{k}+\omega_{o} s_{k}\right) .
$$

Moreover, if $k \in \mathbb{Z}_{n}^{-}$, then $R$ in (6) satisfies $R^{\mathrm{T}} R=(n / 2) I$, and hence, $s_{k}$ and $c_{k}$ form an orthogonal basis for the range space of $M$.

The connectivity matrix $M$ is circulant, has rank two, and admits a simple factorization with an explicit orthogonal basis for the range space. These special properties will be instrumental to our analysis at various different levels. Of particular importance at this point is the reduced-rank property that allows us to obtain a planar system capturing the steady-state behavior of the CPG (1) exactly. The following result shows that a state transformation exists for the CPG so that $n-2$ state variables converge to zero in the steady state, resulting in a planar system for the remaining two states.

Lemma 2.2: Let positive integers $n, k \in \mathbb{Z}$, real scalars $\tau, \omega_{o}, \hbar_{o} \in \mathbb{R}$, and a Lipschitz function $\varphi$ be given. Suppose $k \in \mathbb{Z}_{n}^{-}, \tau=1$, and $\hbar_{o} \neq 0$. Define matrices $M, \Omega$, and $R$ by (5) and (6). Then, every trajectory $x$ of (1) is given by $x=R \xi$ in the steady state for some $\xi$ satisfying

$$
\xi+\dot{\xi}=\Omega R^{\mathrm{T}} \varphi(R \xi) .
$$

Conversely, for each trajectory $\xi$ of (8), the signal $x:=R \xi$ satisfies (1).

Proof: Let $N \in \mathbb{R}^{n \times(n-2)}$ be a matrix such that $N^{\mathrm{T}} R=0$ and $N^{\mathrm{T}} N>0$, and consider the state transformation $x \mapsto(\xi, \eta)$ defined by $\xi:=R^{\dagger} x \in \mathbb{R}^{2}$ and $\eta:=N^{\dagger} x \in \mathbb{R}^{n-2}$. Then, noting that $x=R \xi+N \eta$, the system (1) can be described by

$$
\xi+\dot{\xi}=\Omega R^{\mathrm{T}} \varphi(R \xi+N \eta), \quad \eta+\dot{\eta}=0 .
$$

Hence, in the steady state, we have $\eta=0$ and $x=R \xi$, and $\xi$ satisfies (8).

Remark 2.3: The planar system (8) is equivalent, in the steady state, to the original CPG in (1) with $M$ given in (5). In particular, $x_{e}$ is an equilibrium point of (1) if and only if $\xi_{e}$ is an equilibrium point of (8) and $x_{e}=R \xi_{e}$ holds. Moreover, the CPG (1) has a limit cycle if and only if the planar system (8) does, and the two limit cycle orbits are related by $x=R \xi$.

The key steps to the solution of the CPG synthesis problem can now be summarized as follows. We have shown earlier that a UPO may be given by the CPG (1) with the circulant structure 
(7), which is equivalent to the planar oscillator (8). In Section III, the periodic orbit of the planar oscillator is analyzed in terms of its existence, radial convexity, spatial periodicity, and asymptotically sinusoidal property. In Section IV, the solution to the CPG synthesis problem will be given, using the basic properties of the planar oscillator. Specifically, uniform phase properties of the circulant CPG will be proved first. Once we have an UPO, desired frequency and amplitudes can be achieved by scaling the time axis and state coordinates. Rational phase oscillators (RPOs) with prescribed rational phases can be constructed by projecting the $n$ states of the UPO onto the $m$-dimensional subspace specified by the desired rational phases. For this technique to work, we make sure that the projection of the states onto the $(n-m)$-dimensional orthogonal complement globally converges to zero. These results for the design of UPOs and RPOs will be summarized by Theorem 4.1 in Section IV.

\section{BASIC PLANAR OSCILLATOR}

\section{A. Existence of Periodic Orbit}

The existence of oscillations can be guaranteed by instability of every equilibrium points and boundedness of every trajectories. For planar systems, by the Poincare-Bendixson theorem [25], these conditions, in fact, guarantee that the oscillations are periodic. It turns out that the planar system (8) has a unique equilibrium point if $\left|\omega_{o}\right|$ is sufficiently large, and that the equilibrium point is unstable if $\hbar_{o}$ is positive and small. The boundedness of every trajectory is guaranteed if $\varphi$ is bounded because the transfer function seen by $\varphi$ is stable. Hence, under these conditions, we conclude the existence of a periodic orbit. The result is formally stated later and is proved in the Appendix.

Lemma 3.1: Consider the planar system (8) with $\Omega$ and $R$ in (6) and $\varphi$ satisfying parts 1)-4) of Assumption 2. Let $\gamma \in \mathbb{R}$ be an arbitrary number such that

$$
\begin{aligned}
\max _{\xi \neq 0}\left|\frac{f(\xi)}{g(\xi)}\right| & \leq \gamma \\
f(\xi) & :=\xi^{\mathrm{T}} S R^{\mathrm{T}} \varphi(R \xi), \\
g(\xi) & :=\xi^{\mathrm{T}} R^{\mathrm{T}} \varphi(R \xi), \quad S:=\left[\begin{array}{rr}
0 & 1 \\
-1 & 0
\end{array}\right] .
\end{aligned}
$$

One such $\gamma$ is given by

$$
\gamma:=\min _{h \in \mathbb{Z}_{n}}\left\{\left|\tan \left(\frac{\pi k h}{n}\right)\right|: \frac{k h}{n} \notin \mathbb{Z}\right\} .
$$

If $\left|\omega_{o}\right|>\gamma$ holds, then the origin is the unique equilibrium point. If in addition $0<\hbar_{o}<1$ holds, then the equilibrium point is unstable, and every nontrivial trajectory approaches a periodic orbit.

Remark 3.1: In the aforementioned lemma, the smallest lower bound $\gamma$ on $\left|\omega_{o}\right|$ can be estimated by maximizing $|f(\xi) / g(\xi)|$ through, e.g., an exhaustive search over a plane. This lower bound is potentially conservative. The simple formula in (11) provides a more conservative lower bound that can readily be calculated.

\section{B. Radial Convexity and Spatial Periodicity}

The simplicity of the planar system in (8) allows us to see some structure of the periodic orbit. In particular, the region enclosed by the orbit turns out to be radially convex, i.e., the intersection of the region and any straight line passing through the origin is a convex interval. Moreover, every periodic orbit of (8) is spatially periodic, i.e., the rotation of a periodic orbit by angle $\theta_{k}$ in the two-dimensional state-space yields the same orbit. The key ideas behind this fact are twofold: 1) the planar system (8) is a projection of the original CPG (1) that has the circulant connectivity structure and 2) the $\theta_{k}$-rotation in the $\xi$-plane is equivalent to the circulation in the original $x$-space. The result is summarized later (see Appendix for a proof).

Lemma 3.2: Consider (8) with $\Omega$ and $R$ in (6) and $\varphi$ satisfying parts 1)-4) of Assumption 2. Suppose $0<\hbar_{o}>1$ and $\left|\omega_{o}\right|>\gamma$ for $\gamma$ defined by (11). Then, every periodic orbit can be expressed as

$$
\xi(t)=r(t)\left[\begin{array}{cc}
\cos \theta(t) & \sin \theta(t)
\end{array}\right]^{\mathrm{T}}
$$

for some differentiable functions $r(t)$ and $\theta(t)$ satisfying

$$
\begin{aligned}
r(t) & >0, & r(t) & =r(t-T)=r\left(t \pm \tau_{k}\right) \\
\pm \dot{\theta}(t) & >0, & \theta(t) & =\theta(t-T) \pm 2 \pi=\theta\left(t \pm \tau_{k}\right)-\theta_{k}
\end{aligned}
$$

for all $t$, where $\tau_{k}:=k T / n, T$ is the period, and \pm is taken to be the same sign as $\omega_{0}$.

\section{Asymptotically Sinusoidal Oscillation}

The objective of this section is to address the issue of the wave form. The following result shows that the states of the planar system (8) oscillate in a nearly sinusoidal manner when the number of scalar nonlinearity $n$ is sufficiently large. A proof is given in the Appendix.

Lemma 3.3: Consider the planar oscillator (8) under the condition described in Lemma 3.2. Suppose Assumption 2 holds. Let $\kappa$ be the describing function of $\psi$

$$
\kappa(x):=\frac{1}{r \pi} \int_{0}^{2 \pi} \sin \vartheta \psi(x \sin \vartheta) d \vartheta
$$

and define $\alpha_{o}$ as the unique solution to $\kappa\left(\alpha_{o}\right)=\hbar_{o}$. Then, given any $\epsilon>0$, there exists a sufficiently large $n$ such that every periodic orbit (12) of (8) satisfies

$$
|\dot{r}(t)|<\epsilon, \quad\left|r(t)-\alpha_{o}\right|<\epsilon, \quad\left|\dot{\theta}(t)-\omega_{o}\right|<\epsilon
$$

for all $t$.

Remark 3.2: Clearly, in the limit $\epsilon \rightarrow 0$, we have $r(t)=\alpha_{o}$ and $\theta(t)=\omega t$, resulting in sinusoidal oscillation of $\xi(t)$. Thus, the deviation from the sinusoidal oscillation can be made arbitrarily small by choosing a sufficiently large $n$, the number of nonlinearities $\psi$. In this case, $\alpha_{o}$ and $\omega_{o}$ provide good estimates for the oscillation amplitude and frequency. An implication of this result will be discussed later within the context of optimal control of rectifier systems. 


\section{MAIN RESUlT}

\section{A. Circulant Oscillators}

In this section, we present a basic result that provides a solution to the CPG synthesis problem. To state the result, let us first describe four classes of nonlinear oscillators. Their properties will be given in a theorem later. We will then discuss how a solution to the CPG synthesis problem can be obtained.

Let $m \in \mathbb{Z}, \quad \alpha_{o}, \omega_{o}, \tau \in \mathbb{R}, \quad \alpha, \phi \in \mathbb{R}^{m}$, and a function $\varphi: \mathbb{R}^{n} \rightarrow \mathbb{R}^{n}$ be given. Suppose $m \geq 2$ holds, $\varphi$ satisfies Assumption 2, and $\phi$ satisfies Assumption 1 and (4) for $n, k \in \mathbb{Z}$ and $\eta \in \mathbb{Z}_{n}^{m}$. Define $M, \Omega$, and $R$ by (5) and (6), with $\kappa$ being given by (14), and

$$
\begin{aligned}
\mathcal{A} & :=\operatorname{diag}\left(\alpha_{1}, \ldots, \alpha_{m}\right), \quad \mathcal{M}:=\mathcal{R} \Omega \mathcal{R}^{\mathrm{T}} \\
{\left[\begin{array}{l}
\mathcal{R} \\
\mathcal{S}
\end{array}\right] } & :=\left[\begin{array}{l}
U^{\mathrm{T}} \\
V^{\mathrm{T}}
\end{array}\right] R, \quad \mathcal{L}:=\left[\begin{array}{c}
I \\
\mathcal{S} \mathcal{R}^{\dagger}
\end{array}\right]
\end{aligned}
$$

where $\left[\begin{array}{ll}U & V\end{array}\right] \in \mathbb{R}^{n \times n}$ is a permutation matrix such that $U \in \mathbb{R}^{n \times m}$ and $y_{i}=x_{\eta_{i}}$ holds for $i \in \mathbb{Z}_{m}$ when $y=U^{\mathrm{T}} x$. Let four systems be defined by

$$
\begin{array}{ll}
\xi(t) \in \mathbb{R}^{2}, & \xi+\dot{\xi}=\Omega R^{\mathrm{T}} \varphi(R \xi) \\
x(t) \in \mathbb{R}^{n}, & x+\dot{x}=M \varphi(x) \\
y(t) \in \mathbb{R}^{m}, & y+\dot{y}=\mathcal{M} \mathcal{L}^{\mathrm{T}} \varphi(\mathcal{L} y) \\
z(t) \in \mathbb{R}^{m}, & z+\tau \dot{z}=\mathcal{A M} \mathcal{L}^{\mathrm{T}} \varphi\left(\mathcal{L} \mathcal{A}^{-1} z\right) .
\end{array}
$$

The main result of this paper, given later, states that these systems are oscillators, and describes the properties of their oscillations.

Theorem 4.1: Consider the systems in (17)-(20). Suppose $\left|\omega_{0}\right|>\gamma$ holds for some $\gamma \in \mathbb{R}$ satisfying (9). Then, for each system, there is a unique unstable equilibrium point at the origin, and every nontrivial trajectory converges to a limit cycle. Moreover, the limit cycle trajectories are related by

$$
x=R \xi, \quad y=U^{\mathrm{T}} x, \quad z(t)=\mathcal{A} y\left(\frac{t}{\tau}\right)
$$

and are given by

$$
\begin{aligned}
\xi(t) & =r(t)[\cos \theta(t) \quad \sin \theta(t)]^{\mathrm{T}} \\
x_{i}(t) & =Q\left(\omega t \pm i \theta_{k}\right)=r(t) \sin \left(\theta(t)+i \theta_{k}\right) \\
y_{i}(t) & =Q\left(\omega t \pm \phi_{i}\right)=r(t) \sin \left(\theta(t)+\phi_{i}\right) \\
z_{i}(t) & =\alpha_{i} Q\left(\varpi t \pm \phi_{i}\right)=\alpha_{i} r\left(\frac{t}{\tau}\right) \sin \left(\theta\left(\frac{t}{\tau}\right)+\phi_{i}\right)
\end{aligned}
$$

for some nonzero frequency $\omega$, where $Q$ is a $2 \pi$-periodic function defined by

$$
Q(\omega t):=r(t) \sin \theta(t)
$$

and $r(t)$ and $\theta(t)$ are differentiable functions satisfying (13) and

$$
\lim _{n \rightarrow \infty} r(t)=\alpha_{o}, \quad \lim _{n \rightarrow \infty} \dot{\theta}(t)=\lim _{n \rightarrow \infty} \omega=\omega_{o}
$$

for all $t \in \mathbb{R}$, where \pm is taken to be the same sign as $\omega_{o}$, and

$$
\tau_{k}:=\frac{k T}{n}, \quad T:=\frac{2 \pi}{\omega}, \quad \varpi:=\frac{\omega}{\tau} .
$$

Proof: The properties of the planar system (17) has been proved in the previous section. In particular, its periodic trajectory is given by (21) with $r(t)$ and $\theta(t)$ satisfying the indicated properties. Here, we will prove (22)-(24) by showing the relations among the four systems.

Let us first consider the system (18). Lemmas 2.2 and 3.1 imply that every nontrivial trajectory of (18) approaches a periodic orbit $x=R \xi$. Substituting the definition of $R$ into $x=R \xi$ gives

$$
x_{i}(t)=r(t) \sin \left(\theta(t)+i \theta_{k}\right)
$$

for $i \in \mathbb{Z}_{n}$. Then, we have (22) by noting from Lemma 3.2 that

$$
\begin{aligned}
x_{n}\left(t \pm i \tau_{k}\right)= & r\left(t \pm i \tau_{k}\right) \sin \left(\theta\left(t \pm i \tau_{k}\right)+n \theta_{k}\right) \\
& =r(t) \sin \left(\theta(t)+i \theta_{k}\right)=x_{i}(t)
\end{aligned}
$$

and defining $Q(\omega t):=x_{n}(t)$.

Due to part 2) of Assumption 1, $\mathcal{R}$ has a full column rank, and hence, there exists $L$ such that $\mathcal{S}=L \mathcal{R}$. One such choice is given by $L:=\mathcal{S} \mathcal{R}^{\dagger}$. Define

$$
\left[\begin{array}{l}
y \\
\hat{y}
\end{array}\right]:=\left[\begin{array}{cc}
I & 0 \\
-L & I
\end{array}\right]\left[\begin{array}{l}
U^{\mathrm{T}} \\
V^{\mathrm{T}}
\end{array}\right] x
$$

Then, we have

$$
y+\dot{y}=\mathcal{R} \Omega\left[\begin{array}{l}
\mathcal{R} \\
\mathcal{S}
\end{array}\right]^{\mathrm{T}} \varphi\left[\begin{array}{c}
y \\
\hat{y}+L y
\end{array}\right], \quad \hat{y}+\dot{\hat{y}}=0
$$

which, in the steady state, reduces to (19) since $\hat{y}(t) \rightarrow 0$. The result (23) now follows from (22) by noting $\phi_{i}=\eta_{i} \theta_{k}$.

Let $z(t)$ be defined by scaling the magnitude and time axis of $y(t)$ as $z(t):=\mathcal{A} y(t / \tau)$. Then, it is straightforward to verify that $z(t)$ satisfies (20). From (23), we have

$$
z_{i}(t)=\alpha_{i} y_{i}\left(\frac{t}{\tau}\right)=\alpha_{i} Q\left(\varpi t \pm \phi_{i}\right)
$$

and thus, (24) is proved.

\section{B. Discussion}

The system (18) is the basic circulant UPO that has the phases uniformly spaced over $2 \pi k$. A state coordinate transformation shows that the projection of the state vector of (18) onto the orthogonal complement of the range space of $M$ converges to the origin. The behavior in the remaining two-dimensional range space is captured in the steady state by $\xi(t) \in \mathbb{R}^{2}$ satisfying (17). Thus, the limit cycle of (18) is given by projecting the planar limit cycle of (17) back onto the original $n$-dimensional statespace as $x=R \xi$. The planar system (17) makes it possible to prove the existence and to derive the properties of the periodic orbit, as shown in Section III. The RPO in (19) is derived from the UPO (17) by keeping the $\eta_{i}$ th states that have the specified phases $\phi_{i}$. Finally, any desired amplitudes and frequency can be achieved by scaling the states and the time axis as in (20).

A solution to the CPG synthesis problem can be obtained as follows. For a given phase $\phi \in \mathbb{R}^{m}$, let $n, k \in \mathbb{Z}$ and $\eta \in \mathbb{Z}^{m}$ be such that (4) holds. Choose arbitrary positive numbers $\alpha_{o}, \omega_{o} \in$ $\mathbb{R}$ such that $\omega_{o}>\gamma$. Then, design the RPO (19) and find the frequency $\omega$ and amplitude $Q_{m}:=\max |Q(t)|$ of oscillation 
through a simulation. Set $\tau:=\omega / \varpi$ and $\alpha_{i}:=a_{i} / Q_{m}$, and the scaled RPO (20) provides oscillations $z(t)$ as specified in (3) with $\mathcal{Q}(t):=Q(t) / Q_{m}$.

There is design freedom in the choice of some parameters. As mentioned before, the integers $(n, k, \eta)$ in (4) to express the given phase $\phi$ are not unique, e.g., $n$ and $n_{i}$ can be multiplied by a positive integer without violating (4). The parameter $n$ is the number of nonlinearities $\psi$, and is desired to be small from a complexity consideration. However, if sinusoid-like oscillations are desired, a large $n$ can be chosen to achieve it as stated in Theorem 4.1. This point will be illustrated by numerical examples given later. The parameters $\alpha_{o}$ and $\omega_{o}$ are related to the rate of convergence to the periodic orbit. In particular, the number of cycles required for convergence tends to be larger if $\alpha_{o}$ is smaller and/or $\omega_{o}$ is larger. This can be expected from the system (17) linearized around the origin, whose damping ratio $\zeta$ is given by

$$
\zeta=\frac{1}{\sqrt{1+\mu^{2}}}, \quad \mu:=\frac{\omega_{o}}{\left(1-\kappa\left(\alpha_{o}\right)\right)} .
$$

A small value of $\alpha_{o}$ also has the effect of making the oscillations close to sinusoids because the nonlinearity $\varphi$ can be approximated by a linear gain when the amplitudes are small. These observations on $\alpha_{o}$ and $\omega_{o}$ have been confirmed by numerical simulations, but the details are omitted due to the space limitation.

Example 4.1: We design circulant oscillators with $m=3$ and $\phi=\left[\begin{array}{lll}0 & \pi / 3 & \pi / 2\end{array}\right]^{\mathrm{T}}$. We do not scale the states or the time axis to set specific amplitudes or the frequency. Instead, we set $\alpha_{o}=10$ and $\omega_{o}=1$ to illustrate how close the amplitudes and frequency are to these numbers when $n$ is finite (recall from Theorem 4.1 that they match exactly in the limit $n \rightarrow \infty)$.

We consider the two realizations $(n, k, \eta)$ of the phase specification given in Table I. The RPO in (19) is simulated for each case with random initial states of small magnitudes, and the result is summarized in Figs. 1 and 2, and Table I. The parameter $\gamma$ is the upper bound on the maximum in (9), calculated from (11). It has been verified by gridding the $\xi$-plane that the upper bound values are tight and coincide with the maxima at least up to three digits. The value of $\omega_{o}$ has been chosen so that $\omega_{o}>\gamma$ as required in Theorem 4.1. The parameters $T$ and $Q_{m}$ are the period and amplitude, $\max |Q(t)|$, of the oscillations observed in the simulations. While the amplitudes are close to $\alpha_{o}$ for both cases, the frequency deviation from $\omega_{o}$ tends to be larger if $n$ is smaller. We notice from the figures that the orbits on the $\xi$-plane are clockwise due to $\omega_{o}>0$ and look like polygons with $n$ vertices, confirming the radial convexity and the spatial periodicity proved earlier. As $n$ is increased, the number of vertices gets larger, and the orbit approaches a circle, resulting in sinusoidal oscillations.

\section{Feedback Control For State Pattern Generation}

\section{A. Optimal Regulation of Rectifier Systems}

In this section, we consider a feedback control design problem to achieve dynamic rectification through coordinated oscil-
TABLE I

RATIONAL Phase OSCILlator SimUlation Results $\left(\alpha_{o}=10, \omega_{o}=1\right)$

\begin{tabular}{|c||c|c|c|c|c|c|c|c|}
\hline & $n$ & $k$ & $\eta_{1}$ & $\eta_{2}$ & $\eta_{3}$ & $\gamma$ & $T$ & $Q_{m}$ \\
\hline Case 1 & 6 & 1 & 6 & 1 & 3 & 0.577 & 7.07 & 10.2 \\
\hline Case 2 & 12 & 1 & 12 & 2 & 6 & 0.268 & 6.35 & 10.1 \\
\hline
\end{tabular}
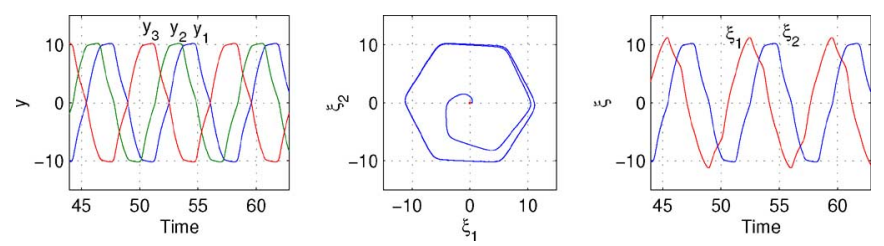

Fig. 1. Case 1: $n=6$.
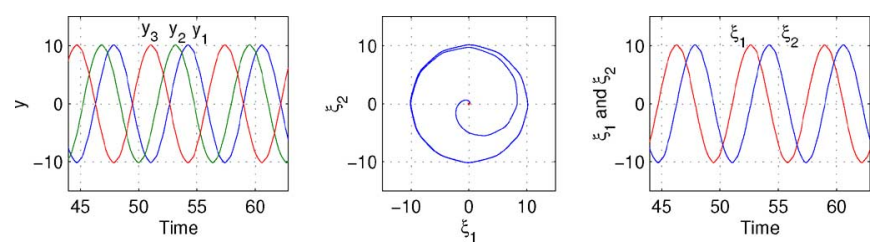

Fig. 2. Case 2: $n=12$.

lations for the following class of nonlinear systems

$$
\dot{z}=u, \quad v+\beta \dot{v}=z^{\mathrm{T}} \Lambda \dot{z}
$$

where $z(t), u(t) \in \mathbb{R}^{m}, \beta>0$, and $\Lambda \in \mathbb{R}^{m \times m}$ with $m$ being any integer greater than or equal to 2 . This system is a generalized version of "the most elementary, and in a limited sense canonical, model" for the rectification of cyclic motions [14]. In particular, periodic inputs $u$ with a proper phase relation can be rectified to result in a nonzero constant output $v$, motivating us to call (25) a rectifier system.

The rectification effect is embedded in the bilinear term $z^{\mathrm{T}} \Lambda \dot{z}$. To discuss, let $\Lambda$ be decomposed into $\Lambda=P+S$ where:

$$
P:=\frac{\left(\Lambda+\Lambda^{\mathrm{T}}\right)}{2}, \quad S:=\frac{\left(\Lambda-\Lambda^{\mathrm{T}}\right)}{2} .
$$

When $z(t)$ is periodic, the integral of $z^{\mathrm{T}} P \dot{z}$ over one cycle is always zero since it is integrable, but that of $z^{\mathrm{T}} S \dot{z}$ can be nonzero. In fact, it can readily be shown that the system (without the $v$ term) is first-bracket controllable (see [14] for a definition) if and only if $\Lambda$ is asymmetric (i.e., $S \neq 0$ ). This property enables set point regulation of $v(t)$ around a desired value through oscillations of $z(t)$.

We would like to design a feedback controller for (25) so that $z(t)$ oscillates in such a way that the variable $v(t)$ is maintained at a desired value $v_{o}$ on average in the steady state. The following theorem provides a basic result to achieve this objective.

Theorem 5.1: Consider the system (25) with positive scalar $\beta \in \mathbb{R}$ and matrix $\Lambda \in \mathbb{R}^{m \times m}$. Let positive numbers $\alpha_{o} \in$ $\mathbb{R}, \omega_{o} \in \mathbb{R}, \quad \tau \in \mathbb{R}, \quad \alpha \in \mathbb{R}^{m}$, and $\phi \in \mathbb{R}^{m}$ be given and define

$$
v_{o}:=j \varpi q^{*} S q, \quad \varpi:=\frac{\omega_{o}}{\tau}, \quad q_{i}:=\frac{\alpha_{o} \alpha_{i} e^{j \phi_{i}}}{\sqrt{2}} .
$$


Suppose $\phi$ satisfies Assumption 1, and is described by (4) for some integers $n, k \in \mathbb{Z}$, and $\eta \in \mathbb{Z}^{m}$. Consider the feedback controller

$$
u=\frac{\mathcal{A M}^{\mathrm{T}} \mathcal{L}^{\mathrm{T}} \varphi\left(\mathcal{L} \mathcal{A}^{-1} z\right)-z}{\tau}
$$

where $\varphi$ is a function satisfying Assumption 2 , and $\mathcal{A}, \mathcal{M}$, and $\mathcal{L}$ are defined in (16). Then, every nontrivial state trajectory $z_{i}(t)$ of the closed-loop system converges to the limit cycle described in (24). Moreover, if $n$ is large enough to guarantee (15) for a small $\epsilon>0$, then $v(t)$ in the steady state is given by ${ }^{1}$

$$
\begin{aligned}
& v(t)=v_{o}+\frac{1}{1+\beta s} \Im\left[\varpi e^{j 2 \theta(t)} q^{\mathrm{T}} P q\right]+O(\epsilon), \\
& \dot{\theta}(t)=\varpi+O(\epsilon)
\end{aligned}
$$

for some function $\theta(t)$.

Proof: The existence and description of the limit cycle are a direct consequence of Theorem 4.1. To prove (29), let us consider the case $\tau=1$ for brevity. The result for the general case can be recovered by noting that $w(t)=w_{1}(t / \tau) / \tau$ where $w_{1}(t):=\left.w(t)\right|_{\tau=1}$ and $w:=z^{\mathrm{T}} \Lambda \dot{z}$. Recall from (24) that $z_{i}=$ $\alpha_{i} r \sin \left(\theta+\phi_{i}\right)$ in the steady state. Taking the time derivative and using trigonometric identities

$$
\begin{aligned}
\alpha_{o}^{2} z^{\mathrm{T}} \Lambda \dot{z}= & (r \dot{r}) \Re\left[q^{*} \Lambda^{\mathrm{T}} q-e^{j 2 \theta} q^{\mathrm{T}} \Lambda q\right] \\
& +\left(r^{2} \dot{\theta}\right) \Im\left[q^{*} \Lambda^{\mathrm{T}} q+e^{j 2 \theta} q^{\mathrm{T}} \Lambda q\right] \\
= & (r \dot{r})\left[q^{*} P q-\Re\left(e^{j 2 \theta} q^{\mathrm{T}} P q\right)\right] \\
& +\left(r^{2} \dot{\theta}\right)\left[j q^{*} S q+\Im\left(e^{j 2 \theta} q^{\mathrm{T}} P q\right)\right]
\end{aligned}
$$

where we noted that

$$
\Re\left[q^{*} \Lambda^{\mathrm{T}} q\right]=q^{*} P q, \quad \Im\left[q^{*} \Lambda^{\mathrm{T}} q\right]=j q^{*} S q, \quad q^{\mathrm{T}} \Lambda q=q^{\mathrm{T}} P q .
$$

Now, the result follows from the fact that $\dot{r}(t), r(t)-\alpha_{o}$, and $\dot{\theta}(t)-\omega_{o}$ are all $O(\epsilon)$ due to (15).

Remark 5.1: When $n$ is sufficiently large, the signal $v(t)$ in the steady state is approximately given by the superposition of the constant bias $v_{o}$ and the ripple, i.e., the second term in (29). If $\Lambda$ is symmetric, then $v_{o}$ is always zero, and hence, $v(t)$ cannot be made to track a nonzero constant on average. Thus, the skew-symmetric part $S$ of $\Lambda$ makes $v(t)$ controllable. On the other hand, the symmetric part $P$ of $\Lambda$ is associated with the size of the ripples in $v(t)$. In particular, if $\Lambda$ is skew symmetric $(P=0)$, then any sinusoidal $z(t)$ leads to constant $v(t)$ without ripples in the steady state.

We now discuss optimal control strategies based on Theorem 5.1. First, let us consider the problem of achieving a given value of $v_{o}$ for $v(t)$ on average with the maximum efficiency. A measure of efficiency may be given by the average value of $\|\dot{z}(t)\|^{2}$ over one cycle since $\dot{z}(t)$ typically represents velocity, and the referred quantity is related to the energy loss due to damping effects. Thus, we formulate the following optimization

\footnotetext{
${ }^{1}$ With a slight abuse of notation, we denote by $G(s) u(t)$ the steady-state output of the transfer function $G(s)$ in response to the input $u(t)$.
}

problem

$$
\max _{z \in \mathbb{P}} \frac{\int_{0}^{T} z(t)^{\mathrm{T}} \Lambda \dot{z}(t) d t}{\int_{0}^{T}\|\dot{z}(t)\|^{2} d t}
$$

where $\mathbb{P}$ is the set of continuously differentiable $T$-periodic functions. In the context of multilink robotic applications that we discuss later, the solution to this problem provides the optimal rhythmic motion leading to a fixed locomotion speed $v_{o}$ using the least energy. The following gives the solution to (30).

Lemma 5.1: The global maximum of (30) is attained by

$$
z_{i}(t)=a_{i} \sin \left(\varpi t+\phi_{i}\right), \quad q_{i}=a_{i} e^{j \phi_{i}}
$$

where $\varpi:=2 \pi / T$ and $q \in \mathbb{C}^{m}$ is the eigenvector of $j S$ corresponding to the largest eigenvalue:

$q=e_{1}, \quad j S e_{i}=\omega_{i} e_{i}, \quad e_{i} \neq 0, \quad \omega_{1} \geq \omega_{2} \geq \cdots \geq \omega_{m}$.

The maximum value of (30) is given by $\omega_{1} / \varpi$.

Proof: Let $z(t)$ be $T$-periodic and consider its Fourier series expansion:

$$
z_{i}(t)=\sum_{k=0}^{\infty} a_{i k} \sin \left(k \varpi t+\phi_{i k}\right)
$$

Using the orthogonality property of sinusoidal basis functions, it is straightforward to verify that

$$
\frac{\int_{0}^{T} z^{\mathrm{T}} \Lambda \dot{z} d t}{\int_{0}^{T}\|\dot{z}(t)\|^{2} d t}=\frac{\sum j \pi k z_{k}^{*} S z_{k}}{\sum \pi \varpi k^{2}\left\|z_{k}\right\|^{2}}=\frac{j \zeta^{*} \mathcal{S} \zeta}{\varpi\|\zeta\|^{2}}
$$

where the summations are over $k=1,2, \ldots$, and $z_{k} \in \mathbb{C}^{m}$ is the vector whose $i$ th entry is $a_{i k} e^{j \phi_{i k}}$ and

$$
\zeta:=\operatorname{col}\left(z_{1}, 2 z_{2}, 3 z_{3}, \ldots\right), \quad \mathcal{S}:=\operatorname{diag}\left(S, \frac{S}{2}, \frac{S}{3}, \ldots\right) .
$$

The maximum of this ratio is attained when $\zeta$ is the eigenvector of $j \mathcal{S}$ corresponding to the largest eigenvalue. Clearly, such $\zeta$ is given by $\zeta=\operatorname{col}\left(e_{1}, 0,0, \ldots\right)$.

Lemma 5.1 shows that the optimal solution $z(t)$ to $(30)$ is sinusoidal with relative amplitudes and phases specified by an eigenvector of the skew-symmetric part of $\Lambda$. Hence, for optimally efficient oscillation, $q$ in (27) should be chosen so that it is the optimal eigenvector described in Lemma 5.1.

Next, we consider the problem of achieving a given $v_{o}$ efficiently with small ripples. In this case, we may formulate an objective function by a weighted sum of the efficiency term $j q^{*} S q$ and the ripple term $\left|q^{\mathrm{T}} P q\right|$ as follows

$$
\min _{q \in \mathbb{C}^{m}} \frac{\sigma\left|q^{\mathrm{T}} P q\right|-j q^{*} S q}{\|q\|^{2}}
$$

where $\sigma \geq 0$ is the weighting parameter. Clearly, if $\sigma=0$, then the solution is given by an eigenvector as described earlier, and if $\sigma$ is sufficiently large, the ripple will be minimized at the expense of efficiency. This is a nonconvex problem and it is difficult to obtain a solution with the guarantee of global optimality. However, it turns out that a local algorithm works well to find a reasonable solution if the initial condition is chosen 
to be the optimally efficient solution given in (31). This point will be illustrated later by a numerical example.

Once we find $q$ by solving one of the aforementioned optimization problems, the optimal relative amplitudes $\hat{\alpha} \in \mathbb{R}^{m}$ and phases $\phi \in \mathbb{R}^{m}$ can be found from $\hat{q}_{i}=\hat{\alpha}_{i} e^{j \phi_{i}}$ where $\hat{q}:=q /\|q\|$. The optimal phases may not be rational in general, and hence, need to be approximated by rational phases so that they can be realized within the circulant oscillator framework. After the rational approximation, the optimization problem should be solved again, over the relative amplitudes $\hat{\alpha}$ with fixed $\phi$, to minimize the approximation error. The next step is to choose the frequency $\varpi$ and amplitude $q_{m}$ such that $\varpi q_{m}^{2}=v_{o} /\left(j \hat{q}^{*} S \hat{q}\right)$. We clearly see the tradeoff between the two as both cannot be small simultaneously.

Finally, a circulant oscillator can be designed by choosing $\alpha_{o}$ and $\omega_{o}$ and setting $\tau:=\omega_{o} / \varpi$ and $\alpha:=\sqrt{2} q_{m}|\hat{q}| / \alpha_{o}$. As mentioned before, convergence to the periodic orbit is faster if $\alpha_{o}$ is larger and $\omega_{o}$ is smaller. Hence, a reasonable choice of $\omega_{o}$ is a value close to the lower bound $\gamma$ in (11). If $\alpha_{o}$ is made larger with $n$ fixed, however, the nonlinearity $\varphi$ tends to saturate, the orbit of $\xi(t)$ becomes polygon-like as in Fig. 1, and the wave form of $z(t)$ deviates from the sinusoid, resulting in more ripples in $v(t)$. Thus, $\alpha_{o}$ can be used as a tuning knob to tradeoff the speed of convergence and the amount of ripples, while keeping $n$ relatively small.

\section{B. Extension to Adaptive Tracking Problem}

From Theorem 5.1, it is clear that if the control $u$ is multiplied by positive constant $\rho$, the rectified velocity becomes $\rho v_{o}$ instead of $v_{o}$. We extend this result for a time varying $\rho(t)$ as stated later.

Lemma 5.2: Consider a nontrivial trajectory $z(t)$ of the closed-loop system in Theorem 5.1 with the feedback control modified to

$$
u=\frac{\rho\left(\mathcal{A} \mathcal{M L}^{\mathrm{T}} \varphi\left(\mathcal{L} \mathcal{A}^{-1} z\right)-z\right)}{\tau}
$$

where $\rho(t)$ is a bounded locally Lipschitz function. Suppose $\left|q^{\mathrm{T}} P q\right| \leq \epsilon$ and $\int_{0}^{\infty} \rho(t) d t=+\infty$. Then, $z(t)$ converges to an invariant manifold on which the following holds:

$$
z(t)^{\mathrm{T}} \Lambda \dot{z}(t)=\rho(t) v_{o}+O(\epsilon) .
$$

Proof: Let $\zeta(t)$ be the trajectory that would result from $\zeta(0)=$ $z(0)$ if $\rho(t) \equiv 1$, and let $\zeta_{o}(t)$ be the periodic orbit to which $\zeta(t)$ converges. Note that, for an arbitrary function $f$, we have

$$
\begin{aligned}
\dot{x} & =\rho f(x), \quad \dot{y}=f(y), \quad x(0)=y(0) \\
\Rightarrow \quad x(t) & =y(\varrho(t)), \quad \varrho(t):=\int_{0}^{t} \rho(t) d t \quad \forall t \geq 0
\end{aligned}
$$

provided the solutions $x$ and $y$ exist and are unique. This fact simply follows from $y_{\varrho}(t):=y(\varrho(t))$ satisfies $\dot{y}_{\varrho}=\rho f\left(y_{\varrho}\right)$ and $y_{\varrho}(0)=x(0)$. Using this fact, we see that $z(t)=\zeta(\varrho(t))$ holds for all $t \geq 0$. Since $\zeta(t)$ converges to $\zeta_{o}(t)$ and $\varrho(t)$ goes to infinity as $t$ increases, $z(t)$ must converge to the invariant manifold $\left\{\zeta_{o}(t) \in \mathbb{R}^{m}: t \in \mathbb{R}\right\}$. In this set, we have

$$
z^{\mathrm{T}} \Lambda \dot{z}-\rho v_{o}=\rho\left(\zeta_{o}(\varrho)^{\mathrm{T}} \Lambda \dot{\zeta}_{o}(\varrho)-v_{o}\right)
$$

and the result now follows from Theorem 5.1 and its proof.

A direct application of the aforementioned lemma is to design a controller to track a time-varying reference velocity $v_{r}(t)$ robustly against possible parameter variations. Specifically, we consider the uncertain rectifier system

$$
\dot{z}=u, \quad v+\beta \dot{v}=\delta z^{\mathrm{T}} \Lambda \dot{z}
$$

where $\beta$ and $\delta$ are uncertain positive constant parameters.

Theorem 5.2: Let $v_{r}(t)$ be a continuously differentiable function and consider the uncertain system (34) and the controller (33) in Lemma 5.2 with $v_{o} \neq 0$ and

$\rho:=a v_{r}+b \dot{v}_{r}, \quad \dot{a}=-a_{o} e v_{r}, \quad \dot{b}=-b_{o} e \dot{v}_{r}, \quad e:=v-v_{r}$

where $a_{o}, b_{o} \in \mathbb{R}$ are positive constants. Suppose $\int_{0}^{\infty} \rho(t) d t=$ $+\infty,\left|q^{\mathrm{T}} P q\right| \leq \epsilon$, and let $T>1 / \epsilon^{2}$. Then, $z(t)$ converges to an invariant manifold on which the following hold:

$$
\frac{1}{T} \int_{0}^{T}\left|v(t)-v_{r}(t)\right|^{2} d t=O\left(\epsilon^{2}\right) .
$$

Proof: Lemma 5.2 guarantees convergence to an invariant manifold on which the error dynamics is governed by

$$
e+\beta \dot{e}=\left(\delta v_{o} a-1\right) v_{r}+\left(\delta v_{o} b-\beta\right) \dot{v}_{r}+p, \quad|p(t)| \leq c \epsilon
$$

for some constant $c>0$, where the bound on the magnitude of $p:=z^{\mathrm{T}} \Lambda \dot{z}-\rho v_{o}$ comes from Lemma 5.2. Define the Lyapunov function

$$
V:=\beta e^{2}+\frac{\left(\delta v_{o} a-1\right)^{2}}{\left(\delta v_{o} a_{o}\right)}+\frac{\left(\delta v_{o} b-\beta\right)^{2}}{\left(\delta v_{o} b_{o}\right)} .
$$

Then, its time derivative along the trajectory is given by:

$$
\begin{aligned}
\frac{\dot{V}}{2} & =\beta e \dot{e}+\left(\delta v_{o} a-1\right)\left(\frac{\dot{a}}{a_{o}}\right)+\left(\delta v_{o} b-\beta\right)\left(\frac{\dot{b}}{b_{o}}\right) \\
& =\beta e \dot{e}-e(e+\beta \dot{e}+p)=-e(e+p) .
\end{aligned}
$$

Noting that $e^{2} \leq p^{2}+2 e(e+p)$, we have

$$
\frac{1}{T} \int_{0}^{T} e^{2} d t \leq c^{2} \epsilon^{2}+\frac{V(0)-V(T)}{T} \leq\left(c^{2}+V(0)\right) \epsilon^{2} .
$$

Remark 5.2: In Theorem 5.2, the tracking is practically achieved under the persistent excitation (PE) condition $\int_{0}^{\infty} \rho(t)$ $d t=+\infty$ which is not a priori guaranteed. However, by an approximate analysis, we expect that the PE condition is satisfied under the following two conditions: 1) the controller is run with $\rho(t)=1$ for a sufficiently long time before turning on the adaptive update law for $a$ and $b$; and 2) $v_{o}$ is positive and $v_{r}(t)$ is bounded and satisfies $\int_{0}^{\infty} v_{r}(t) d t=+\infty$ and $\lim _{t \rightarrow \infty} v_{r}(t) \neq 0$.

Condition 1 ensures that $z(t)$ converges to the invariant manifold regardless of the values of $\beta$ and $\delta$. If $z(t)$ is exactly on the manifold (limit cycle orbit), condition 2 then (roughly) guarantees that $\rho$ satisfies the PE condition as explained later. This means that $z(t)$ goes around in the right direction on the orbit, 


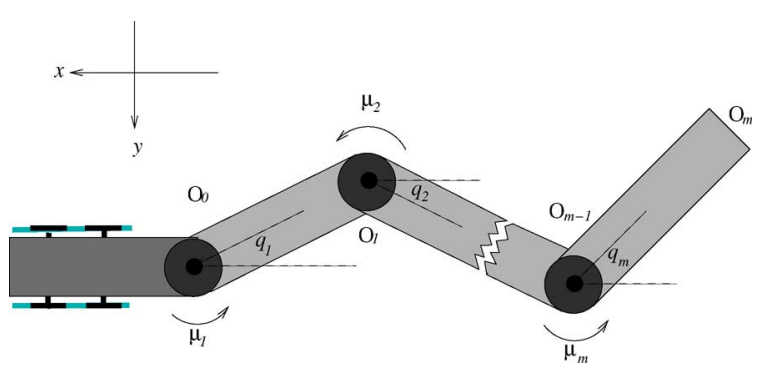

Fig. 3. Snakelike mechanical system (topview).

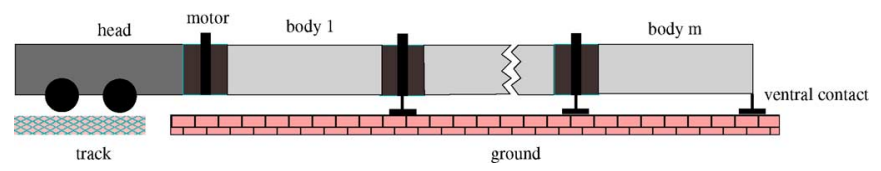

Fig. 4. Snakelike mechanical system (sideview).

and hence, by continuity of solutions of differential equations, we expect that the PE condition for $\rho$ is satisfied even when $z(t)$ is slightly perturbed from the orbit.

To see that $\rho$ is expected to satisfy the PE condition under condition 2, first note that if $z(t)$ is on the orbit and $\epsilon=0$, then the Lyapunov argument in the aforementioned proof guarantees convergence of $e(t)$ to zero. If $e(t) \equiv 0$, then $a(t)$ and $b(t)$ are constant, and

$$
\left(\delta v_{o} a-1\right) v_{r}(t)+\left(\delta v_{o} b-1\right) \dot{v}_{r}(t) \equiv 0 .
$$

We now claim that $a=1 /\left(\delta v_{o}\right)$. To see this, suppose this is not true. If $\delta v_{o} b \neq 1$, then $v_{r}(t)$ is an exponential function, contradicting condition 2. Hence, $\delta v_{o} b=1$, but this again leads to a contradiction $\left(\delta v_{o} a-1\right) v_{r}=0$. Hence, we have $a=1 /\left(\delta v_{o}\right)>0$. Now, as $T \rightarrow \infty$,

$$
\int_{0}^{T} \rho(t) d t=a \int_{0}^{T} v_{r}(t) d t+b\left(v_{r}(T)-v_{r}(0)\right) \rightarrow+\infty
$$

and thus, $\rho$ satisfies the PE condition.

\section{Gait Control of a Snakelike System}

In this section, we will illustrate the utility of Theorems 5.1 and 5.2 through an application to the gait control of a multilink snakelike system shown in Figs. 3 and 4. The system consists of a chain of $m+1$ rigid links, which we call the head, body $1, \ldots$, body $m$. The $i$ th link is of length $\ell_{i}$ and mass $m_{i}$ An actuator is mounted at every joint $O_{i}$ to generate torque $\mu_{i}$. The clockwise angular displacement of body $i$ relative to the positive $x$-axis is denoted by $z_{i}$. The Cartesian coordinate of joint $O_{i}$ is denoted by $\left(x_{i}, y_{i}\right)$. The head is constrained to move on a straight track along the $x$-axis, and we take the origin of the $x$-axis so that $x_{o}=0$. The propelling force is to be generated by the friction between the ventral contacts between the joints and the ground. The objective is to design a feedback controller to generate torques $\mu(t) \in \mathbb{R}^{m}$ based on the measurements of $z(t) \in \mathbb{R}^{m}$ so that the head velocity $v:=\dot{x}_{o}$ is maintained at a given value $v_{o}$.
TABLE II

FiXed PARAMETERS OF THE SNAKELIKE SYSTEM AND CONTROLLER

\begin{tabular}{|c|c|c|c|c|c|c|}
\hline$v_{o}$ & $m_{o}$ & $c_{o}$ & $c$ & $\ell$ & $m$ & $n$ \\
\hline 1 & 0.01 & 1 & 1 & 1 & 4 & 8 \\
\hline$k$ & $\gamma$ & $\varpi$ & $\alpha_{o}$ & $\omega_{o}$ & $\tau$ \\
\hline 1 & 0.414 & 100 & 1 & 0.5 & 0.005 \\
\hline
\end{tabular}

TABLE III

OPTIMIZED PARAMETERS OF THE CONTROLLER: $\hat{q}$ IS OPTIMIZED FOR CASES 1

\begin{tabular}{|c|c|c|c|c|c|c|}
\hline & $j \hat{q}^{*} S \hat{q}$ & \multicolumn{2}{|c|}{$\left|\hat{q}^{\top} P \hat{q}\right|$} & $q_{m}$ & $\hat{\alpha}_{1}$ & $\hat{\alpha}_{2}$ \\
\hline Case 1 & 1.207 & \multicolumn{2}{|c|}{0.854} & 0.091 & 0.5 & 0.5 \\
\hline Case 2 & \multicolumn{2}{|c|}{0.923} & $2.9 \times 10^{-9}$ & 0.104 & 0.707 & 0.360 \\
\hline Case 3 & 0.886 & \multicolumn{2}{|c|}{$1.7 \times 10^{-7}$} & 0.106 & 0.726 & 0.446 \\
\hline$\hat{\alpha}_{3}$ & $\hat{\alpha}_{4}$ & \multirow{2}{*}{$\frac{\phi_{1}}{0}$} & $\phi_{2}$ & $\phi_{3}$ & \multicolumn{2}{|c|}{$\phi_{4}$} \\
\hline 0.5 & \multirow{2}{*}{$\begin{array}{c}0.5 \\
0.505\end{array}$} & & $-45^{\circ}$ & $-90^{\circ}$ & \multicolumn{2}{|c|}{$-135^{\circ}$} \\
\hline 0.341 & & $\frac{0}{31.7^{\circ}}$ & $-45.0^{\circ}$ & \multirow{2}{*}{$\frac{-91.7^{\circ}}{-90^{\circ}}$} & \multirow{2}{*}{\multicolumn{2}{|c|}{$\frac{-134.2^{\circ}}{-135^{\circ}}$}} \\
\hline 0.354 & $\begin{array}{l}0.505 \\
0.386\end{array}$ & $45^{\circ}$ & $-45^{\circ}$ & & & \\
\hline
\end{tabular}
AND 2, AND $\hat{\alpha}$ IS OPTIMIZED FOR CASE 3

The friction force at the $i$ th contact in the direction tangent (normal) to the body is assumed proportional to the body velocity in that direction with coefficient $c_{t_{i}}\left(c_{n_{i}}\right)$ [26]. It has been observed [27] that the directional property $c_{n_{i}} \gg c_{t_{i}}$ is crucial for a gait of snake locomotion. For simplicity, we assume, for the body, that the tangential frictions are zero and the normal frictions are uniform $\left(c_{t_{i}}=0\right.$ and $c_{n_{i}}=c$ for $\left.i \in \mathbb{Z}_{m}\right)$. We also assume that the head is much heavier than the body $\left(m_{o} \gg m_{i}\right.$ for $i \in \mathbb{Z}_{m}$ ), and hence, the body mass can be ignored. In this case, the torque balance equation for the "tail" consisting of bodies $i \in \mathbb{Z}_{m}$ is given by

$$
A(z) \dot{z}+B(z) v=\mu
$$

for appropriately defined continuous functions $A(z) \in \mathbb{R}^{m \times m}$ and $B(z) \in \mathbb{R}^{m \times 1}$ where $A(0)$ is nonsingular. The equation of motion for the head is given by

$$
m_{o} \dot{v}=-\left(c_{o}+c\|s\|^{2}\right) v-c \ell s^{\mathrm{T}} C \dot{z}
$$

where $s \in \mathbb{R}^{m}$ and $C \in \mathbb{R}^{m \times m}$ are defined by their entries; $s_{i}:=\sin z_{i}, c_{i j}=\cos \left(z_{i}-z_{j}\right)$ for $i \geq j$, and $c_{i j}=0$ for $i<j$. Thus, the motion of the multilink system is described by (36) and (37).

To design a controller within the framework of Theorem 5.1, we shall first introduce the feedback linearization $\tau=A(q) u+$ $B(q) v$ that gives $\dot{z}=u$ where $u(t) \in \mathbb{R}^{m}$ is the new control variable. This approach is valid in the neighborhood of the origin $z=0$ because $A(0)$ is nonsingular, and hence, so is $A(z)$ for sufficiently small $\|z\|$. We shall design a circulant CPG that produces oscillations of $z$ with sufficiently small amplitudes. In this case, the system can be approximated by the bilinear model (25) with $\beta:=m_{o} / c_{o}$ and $\Lambda$ is the lower triangular matrix with uniform nonzero entries $-c \ell / c_{o}$.

We design optimal controllers for the snakelike system by solving the problem in (32). The plant parameters and some of the controller parameters are fixed as in Table II. The problem (32) is solved for three cases as summarized in Table III, where $\hat{q}:=q /\|q\|, \hat{q}_{i}=\hat{\alpha}_{i} e^{j \phi_{i}}$, and $q_{m}:=\sqrt{v_{o} /\left(j \varpi \hat{q}^{*} S \hat{q}\right)}$. In case $1, q$ is optimized with $\sigma=0$ for maximum efficiency. Note that the optimally efficient phases are rational because $S$ is a 

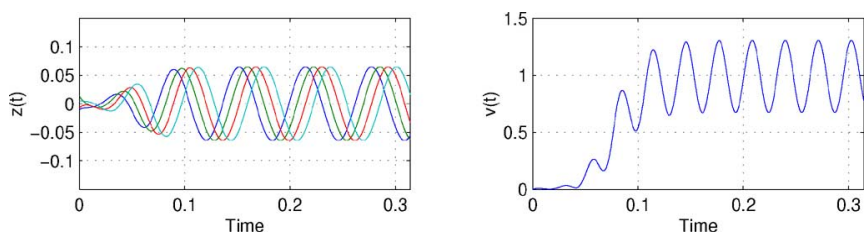

Fig. 5. Locomotion with optimal efficiency (case 1).
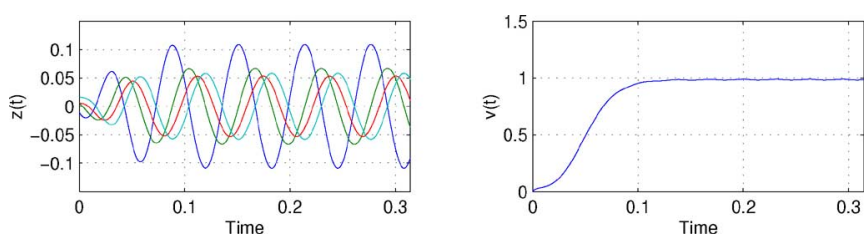

Fig. 6. Locomotion with small ripples (case 3).

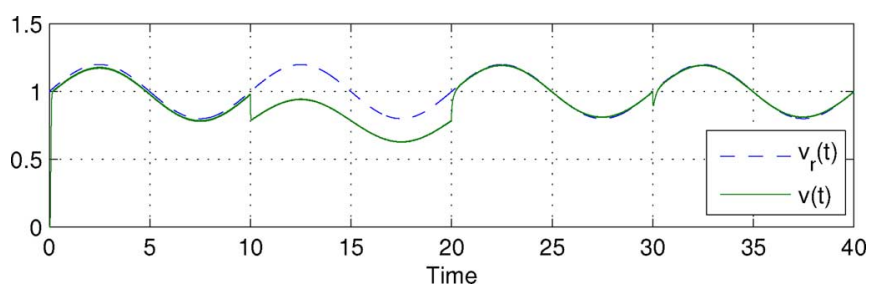

Fig. 7. Profile of the adaptive tracking behavior (the adaptive law is on after $t=20)$.

skew-circulant matrix. In case $2, q$ is optimized with $\sigma=1$ to achieve reasonable efficiency with small ripples. The ripple term $\left|\hat{q}^{\mathrm{T}} P \hat{q}\right|$ has been made essentially zero with a small reduction in the efficiency term $\hat{q}^{*} S \hat{q}$. However, the resulting phases are irrational. In case $3, \hat{\alpha}$ is optimized with $\sigma=1$ while $\phi$ is fixed to rational approximations of the phases obtained in case 2 . For cases 2 and 3, the optimization problem (32) is solved using the command "fminsearch" in Matlab, where the initial conditions are chosen to be the results of cases 1 and 2, respectively.

Two controllers are designed for cases 1 and 3 based on Theorem 5.1. Figs. 5 and 6 show simulations of the nonlinear closed-loop system (37) and (20) with small random initial states. The optimally efficient controller (case 1) achieves the desired velocity $v_{o}=1$ on average. Large ripples are observed in $v(t)$ as predicted from the large value of $\left|\hat{q}^{\mathrm{T}} P \hat{q}\right|$. The controller in case 3 suppresses the ripples by properly adjusting the amplitudes and phases of $z(t)$. In these designs, the amplitudes of $z(t)$ are made small so that the bilinear model (25) provides a reasonable approximation of the original system (37). If, however, the frequency $\varpi$ is chosen to be small, then the amplitudes get larger, the bilinear model becomes invalid, and ripples appear in $v(t)$ even for case 3 .

We now add the adaptive law in Theorem 5.2 to the controller designed in case 3 earlier. With $v_{r}(t)=1+0.2 \sin (0.2 \pi t)$, a simulation result is plotted in Fig. 7, where the parameter values are as specified before except for $(\beta, \delta)$ that are varied as indicated in Table IV. For the first $10 \mathrm{~s}$, the values of $(\beta, \delta)$ are the same as those used for the controller design, the adaptive
TABLE IV

Simulation CONDITION FOR ADAPTIVE CPG CONTROL IN FIG. 7

\begin{tabular}{|c||c|c|c|c|}
\hline time & $0-10$ & $10-20$ & $20-30$ & $30-40$ \\
\hline adaptive law & off & off & on & on \\
\hline$(\beta, \delta)$ & $(0.01,1)$ & $(0.012,0.8)$ & $(0.012,0.8)$ & $(0.011,0.7)$ \\
\hline
\end{tabular}

law (35) is off, and the following constant $a$ and $b$ are used to specify $\rho(t)$ :

$$
a:=\frac{1}{\left(\delta v_{o}\right)}=100, \quad b:=\frac{\beta}{\left(\delta v_{o}\right)}=100 .
$$

At $t=10$, the parameters $(\beta, \delta)$ change their values and the tracking performance degrades significantly. When the adaptive law for $a$ and $b$ is turned on at $t=20$, the tracking error quickly converges to zero. Good tracking performance is maintained even after another step change of the parameters at $t=30$.

\section{CONCLUSION}

We have developed a method for designing oscillators with prescribed frequency, amplitudes, and rational phases. Our approach is based on the UPOs achieved by circulant connectivity architectures, and their projections onto appropriate subspaces. It is proved that every nontrivial trajectory converges exactly to a limit cycle with a desired oscillation profile. This is in contrast with approximate methods such as the harmonic balance (see, e.g., [24], [28]) that would not provide this type of theoretical guarantees. The uniqueness of the periodic orbit has not been established, but no multiple orbits have been observed in numerical simulations. Our design method will be useful for constructing local segmental oscillators within a coupled oscillator framework [18], [29].

In the second half of this paper, we have developed a method for designing feedback controllers for a class of bilinear rectifier systems. The control objective is to maintain the rectified signal around a given constant value in the steady state. The key idea is to design the feedback law so that the closed-loop system becomes a circulant oscillator with a prescribed oscillation profile. The general numerical method described in [30] may be used to find the optimal oscillation profile, but our analytical result provides more insight into the bilinear rectification mechanism. In particular, the phases and amplitudes of the optimally efficient rectification are characterized in terms of an eigenvector of $j\left(\Lambda-\Lambda^{\mathrm{T}}\right)$, where $z^{\mathrm{T}} \Lambda \dot{z}$ is the rectifying dynamics in (25). We have also shown that the ripples in the rectified signal arise from the symmetric part of $\Lambda$, and discussed how to suppress the ripples if desired.

To close this paper, let us discuss applications of our results and some open issues for further research. An obvious application would be toward robotic locomotion systems. With the proposed control design method, difficulty of implementation may become an issue for some particular cases as the circulant structure could require distant components be directly wired. Such potential difficulty may be avoided by further study and adoption of biological mechanisms. Specifically, many biological control systems consist of interconnected segmental 
oscillators. For instance, the CPG of leeches is a chain of segmental oscillators with intersegmental connections within some neighboring oscillators only [31]. If this coupled oscillator framework is adopted, our circulant oscillator can be used for the design of segmental oscillators where the neuronal connections can be all-to-all. An important open problem then is how to make structured interconnections of the segmental oscillators to achieve desired phase relations among the segmental oscillators. Such an approach may allow for realization of various coexisting gait patterns, which is not directly possible by the method proposed here. See [32], [33] for some prior attempts at realizations of legged robots with multiple gaits.

\section{APPENDIX I}

\section{PRELIMINARY RESULTS}

This section presents three lemmas which will be used in the proofs of other lemmas. Specifically, the first two lead to the proof of Lemma 3.1, while the latter two are used to prove Lemma 3.2.

Lemma I.I (Rearrangement Inequality, [34]): Let $x, y \in \mathbb{R}^{n}$ be given. Suppose $x_{i+1} \geq x_{i}$ and $y_{i+1} \geq y_{i}$ hold for all $i=$ $1,2, \ldots, n-1$. Define $K \in \mathbb{R}^{n \times n}$ by $K_{i j}=1$ if $i+j=n+1$ and $K_{i j}=0$ otherwise. Then

$$
x^{\mathrm{T}} y \geq x^{\mathrm{T}} P y \geq x^{\mathrm{T}} K y
$$

holds for any permutation matrix $P$.

Lemma I.2: Consider the planar system (8) where $\Omega$ and $R$ are defined by (6) and $\varphi$ satisfies parts 2)-4) of Assumption 2 . The origin is the unique equilibrium point of (8) if $\left|\omega_{o}\right|>\gamma$ holds for $\gamma$ satisfying (9).

Proof: Note that $\xi$ is an equilibrium point of (8) if and only if $\xi=\Omega R^{\mathrm{T}} \varphi(R \xi)$. This condition implies

$$
\xi^{\mathrm{T}} S \Omega R^{\mathrm{T}} \varphi(R \xi)=\frac{2}{n \hbar}\left(f(\xi)+\omega_{o} g(\xi)\right)=0 .
$$

Noting that $\psi$ is strictly in the first and third quadrants due to parts 2)-4) of Assumption 2, we have $g(\xi)>0$ for any nonzero $\xi$. Hence, if $\left|\omega_{o}\right|>\gamma$, then this condition cannot hold unless $\xi=0$, implying that the origin is the unique equilibrium point.

To show that a choice of $\gamma$ is given by (11), it suffices to prove

$$
\left|\frac{f(\xi)}{g(\xi)}\right| \leq\left|\frac{1-\cos \left(h \theta_{k}\right)}{\sin \left(h \theta_{k}\right)}\right| \quad \forall \xi \neq 0
$$

for any integer $h$ satisfying $\sin \left(h \theta_{k}\right) \neq 0$. Let $\xi \in \mathbb{R}^{2}$ be expressed in the polar form $\xi_{1}=r \cos \theta$ and $\xi_{2}=r \sin \theta$. Then, we have

$$
\begin{aligned}
f(\xi) & =\sum_{i=1}^{n} f_{i}(\xi), \quad g(\xi)=\sum_{i=1}^{n} g_{i}(\xi) \\
f_{i}(\xi) & =r \cos \left(i \theta_{k}+\theta\right) \psi\left(r \sin \left(i \theta_{k}+\theta\right)\right) \\
g_{i}(\xi) & =r \sin \left(i \theta_{k}+\theta\right) \psi\left(r \sin \left(i \theta_{k}+\theta\right)\right) .
\end{aligned}
$$

Note that

$$
\begin{aligned}
& \cos \left(h \theta_{k}\right) g(\xi) \pm \sin \left(h \theta_{k}\right) f(\xi)=\sum_{i=1}^{n} \sigma_{i \pm h} \psi\left(\sigma_{i}\right) \\
& \leq \sum_{i=1}^{n} \sigma_{i} \psi\left(\sigma_{i}\right)=g(\xi), \quad \sigma_{i}:=r \sin \left(i \theta_{k}+\theta\right)
\end{aligned}
$$

where we used the rearrangement inequality (Lemma I.1) based on the following facts.

1) For any integer $h,\left(\sigma_{1 \pm h}, \sigma_{2 \pm h}, \ldots, \sigma_{n \pm h}\right)$ is a permutation of $\left(\sigma_{1}, \sigma_{2}, \ldots, \sigma_{n}\right)$ due to the $n$-periodicity $\sigma_{i+n}=$ $\sigma_{i}$.

2) $\left(\sigma_{1}, \sigma_{2}, \ldots, \sigma_{n}\right)$ and $\left(\psi\left(\sigma_{1}\right), \psi\left(\sigma_{2}\right), \ldots, \psi\left(\sigma_{n}\right)\right)$ are arranged in the same order in the sense that the permutation matrix that sorts the former in the descending/ascending order will sort the latter in the descending/ascending order as well, because $\psi$ is monotonically nondecreasing.

Now we have $\left|\sin \left(h \theta_{k}\right) f(\xi)\right| \leq\left(1-\cos \left(h \theta_{k}\right)\right) g(\xi)$ for any $\xi \in \mathbb{R}^{2}$ and integer $h$, from which the result simply follows.

Lemma I.3: Let vectors $x, \hat{x} \in \mathbb{R}^{n}$ and $\xi, \hat{\xi} \in \mathbb{R}^{2}$ be given. Suppose $x=R \xi$ and $\hat{x}=R \hat{\xi}$ hold where $R$ is defined in (6). Then

$$
\begin{gathered}
\hat{x}=J x \Leftrightarrow \hat{\xi}=\Phi \xi \\
J:=O(1,0, \ldots, 0), \quad \Phi:=\left[\begin{array}{cc}
\cos \theta_{k} & -\sin \theta_{k} \\
\sin \theta_{k} & \cos \theta_{k}
\end{array}\right] .
\end{gathered}
$$

If $\xi(t)$ is a periodic orbit of (8), then $\hat{\xi}(t):=\Phi \xi(t)$ is also a periodic orbit of (8).

Proof: Using the identities

$$
R^{\mathrm{T}} J=\Phi R^{\mathrm{T}}, \quad R \Phi=J R
$$

we have

$$
\begin{aligned}
& \hat{x}=J x \Rightarrow \hat{\xi}=R^{\dagger} \hat{x}=\left(\frac{2}{n}\right) R^{\mathrm{T}} J R \xi=\Phi \xi \\
& \hat{\xi}=\Phi \xi \Rightarrow \hat{x}=R \hat{\xi}=R \Phi \xi=J R \xi=J x .
\end{aligned}
$$

Thus, the equivalence holds. To prove the latter statement, recall that if $\xi(t)$ is a periodic orbit of (8), then $x(t):=R \xi(t)$ is a periodic orbit of (1). This, in turn, implies that $\hat{x}(t):=J x(t)$ is a periodic orbit of (1). To see this, multiply (1) by $J$ from left to get

$$
J x+J \dot{x}=M \varphi(J x)
$$

where we noted that $J M=M J$ due to $J, M \in \mathbb{O}$, and $J \varphi(x)=$ $\varphi(J x)$ because $\varphi$ is diagonal and $J$ is a permutation. Thus, $\hat{x}(t)$ is also a periodic orbit of (1). It now follows that $\hat{\xi}(t):=$ $R^{\dagger} \hat{x}(t)=\Phi \xi(t)$ is a periodic orbit of (8).

\section{APPENDIX II}

\section{PROOFS}

Proof of Lemma 2.1: Noting that

$$
\begin{array}{ll}
n \theta_{k}=2 \pi k, & \cos \left((n-i) \theta_{k}\right)=\cos \left(-i \theta_{k}\right) \\
& \sin \left((n-i) \theta_{k}\right)=\sin \left(-i \theta_{k}\right)
\end{array}
$$


we see that the $(i, j)$ entry of $O\left(c_{k}\right)$ and $O\left(s_{k}\right)$ are given by $\cos \left((j-i) \theta_{k}\right)$ and $\sin \left((j-i) \theta_{k}\right)$, respectively. It can then be verified from trigonometric identities that

$$
O\left(c_{k}\right)=c_{k} c_{k}^{\mathrm{T}}+s_{k} s_{k}^{\mathrm{T}}, \quad O\left(s_{k}\right)=c_{k} s_{k}^{\mathrm{T}}-s_{k} c_{k}^{\mathrm{T}} .
$$

The result (7) now follows from linearity of $O(\cdot)$.

To show the latter part, define

$$
e_{k}:=\left[\begin{array}{llll}
e^{j \theta_{k}} & e^{j 2 \theta_{k}} & \cdots & e^{j n \theta_{k}}
\end{array}\right]^{\mathrm{T}}, \quad z_{k}:=e^{j 2 \theta_{k}}
$$

and note that

$$
\begin{gathered}
z_{k} \neq 1, \quad z_{k}^{n}=1, \quad\left\|e_{k}\right\|^{2}=n, \\
e_{k}^{\mathrm{T}} e_{k}=\sum_{i=1}^{n} z_{k}^{i}=\frac{z_{k}\left(1-z_{k}^{n}\right)}{1-z_{k}}=0 .
\end{gathered}
$$

Then, the result follows from

$$
\begin{aligned}
& {\left[\begin{array}{ll}
s_{k} & c_{k}
\end{array}\right]^{\mathrm{T}}\left[\begin{array}{ll}
s_{k} & c_{k}
\end{array}\right] } \\
&=\frac{1}{2}\left[\begin{array}{cc}
\left\|e_{k}\right\|^{2}+\Re\left(e_{k}^{\mathrm{T}} e_{k}\right) & \Im\left(e_{k}^{\mathrm{T}} e_{k}\right) \\
\Im\left(e_{k}^{\mathrm{T}} e_{k}\right) & \left\|e_{k}\right\|^{2}-\Re\left(e_{k}^{\mathrm{T}} e_{k}\right)
\end{array}\right]
\end{aligned}
$$

where $\Re(\cdot)$ and $\Im(\cdot)$ denote the real and imaginary parts, respectively.

Proof of Lemma 3.1: By Lemma I.2, the system (8) has a unique equilibrium point $\xi=0$. The linearized system around the origin is given by

$$
\xi+\dot{\xi}=\frac{\varphi^{\prime}(0)}{\hbar_{o}}\left[\begin{array}{cc}
1 & -\omega_{o} \\
\omega_{o} & 1
\end{array}\right] \xi
$$

where we noted that $R^{\mathrm{T}} R=(n / 2) I$. Since $0<\hbar_{o}<\psi^{\prime}(0)$, the matrix $\varphi^{\prime}(0)(n / 2) \Omega-I$ has eigenvalues in the open right half plane. Therefore, the equilibrium point $\xi=0$ is unstable.

On the other hand, the trajectory $\xi(t)$ can be viewed as the output of a stable transfer function $\Omega /(1+s)$ with input $u:=R^{\mathrm{T}} \varphi(R \xi)$. Since $u$ is always bounded due to boundedness of $\varphi$, every trajectory is bounded. Hence, by [25, Lemma 3.1], a nontrivial trajectory converges to a positive limit set, which contains no equilibrium point because there is a single unstable equilibrium point. Hence, by the Poincare-Bendixson theorem [25, Th. 7.1, p. 290], the positive limit set is a periodic orbit.

Proof of Lemma 3.2: By [25, Corollary 7.1], the closed periodic orbit $\xi(t)$ must encircle the equilibrium point (the origin). Hence, it can be given by (12) for some periodic function $r(t)>0$ (let $T$ be the period) and for some function such that $\theta(t+T)=\theta(t) \pm 2 \pi$ where the sign of \pm is negative/positive if the orbital trajectory is clockwise/counterclockwise. To determine the direction of the orbit, note that

$$
r^{2} \dot{\theta}=\xi^{\mathrm{T}} S \dot{\xi}=\frac{2}{n \hbar_{o}}\left(f(\xi)+\omega_{o} g(\xi)\right)
$$

where $f(\xi), g(\xi)$, and $S$ are defined in (10). Since $\hbar_{O}>0$, $g(\xi)>0$, and $\left|\omega_{o} g(\xi)\right| \geq|f(\xi)|$ by Lemma I.2, we see that $\dot{\theta}(t)$ does not change its sign over time, and the sign of $\dot{\theta}(t)$ is same as the sign of $\omega_{o}$.

Fix a periodic orbit $\xi(t)$ of the planar system (8). From Lemma I.3, $\hat{\xi}(t):=\Phi \xi(t)$ is also a periodic orbit. We claim that these orbits are identical, i.e, $\mathbb{X}=\hat{\mathbb{X}}$ where

$$
\mathbb{X}:=\{\xi(t): t \in \mathbb{R}\}, \quad \hat{\mathbb{X}}:=\{\hat{\xi}(t): t \in \mathbb{R}\} .
$$

To see this, first note that the orbit $\hat{\mathbb{X}}$ is obtained by rotating the orbit $\mathbb{X}$ by $\theta_{k}$ around the origin. On the other hand, each of the orbits must encircle the origin, and the angle $\theta$ of $\xi$ in (12) is a monotonic function of time as shown earlier. Hence, the two orbits can be characterized as

$$
\begin{aligned}
& \mathbb{X}=\left\{\rho(\vartheta)\left[\begin{array}{ll}
\cos \vartheta & \sin \vartheta
\end{array}\right]^{\mathrm{T}}: \vartheta \in \mathbb{R}\right\} \\
& \hat{\mathbb{X}}=\left\{\rho\left(\vartheta+\theta_{k}\right)\left[\begin{array}{ll}
\cos \vartheta & \sin \vartheta
\end{array}\right]^{\mathrm{T}}: \vartheta \in \mathbb{R}\right\}
\end{aligned}
$$

for some positive continuous $2 \pi$-periodic function $\rho$. Since $\rho$ is continuous and periodic, there exists $\vartheta_{0}$ such that $\rho\left(\vartheta_{0}\right)=$ $\rho\left(\vartheta_{o}+\theta_{k}\right)$. This means that there is an intersection of the two orbits $\mathbb{X}$ and $\hat{\mathbb{X}}$, which, in turn, implies that the two orbits must be identical.

We now see that there exists $\tau \in \mathbb{R}$ such that

$$
\begin{aligned}
& |\tau|<T, \quad \xi(t)=\hat{\xi}(t+\tau)=\Phi \xi(t+\tau) \quad \forall t \\
\Rightarrow & r(t)=r(t+\tau), \quad \theta(t)=\theta(t+\tau)+\theta_{k} \quad \forall t \\
\Rightarrow & \theta(t+n \tau)=\theta(t)-n \theta_{k}=\theta(t)-2 \pi k \quad \forall t .
\end{aligned}
$$

Since $\theta(t)$ is monotonic and it takes $T$ seconds for $\theta(t)$ to travel the distance of $2 \pi$, we have $n \tau= \pm k T$ where \pm is taken opposite to the sign of $\omega_{o}$ due to (13). The result follows by defining $\tau_{k}:=|\tau|$.

Proof of Lemma 3.3: The system (8) can be described in the polar coordinate (12) as

$$
\begin{aligned}
r \dot{r} & =\xi^{\mathrm{T}} \dot{\xi}=\frac{2}{n \hbar_{o}}\left(g-\omega_{o} f\right)-r^{2}, \\
r^{2} \dot{\theta} & =\xi^{\mathrm{T}} S \dot{\xi}=\frac{2}{n \hbar_{o}}\left(f+\omega_{o} g\right)
\end{aligned}
$$

where $f, g$, and $S$ are defined in (10). The functions $f$ and $g$ can also be described by (38). With a slight abuse of notation, we write $f(r, \theta)$ to mean $f\left(\left[\begin{array}{ll}r \cos \theta & r \sin \theta\end{array}\right]^{\mathrm{T}}\right)$, and similarly for $g(r, \theta)$. We will first prove the following properties

$$
\begin{aligned}
|f(r, \theta)| & \leq 4 \pi k r^{2} \\
|g(r, \theta)-g(r, \vartheta)| & \leq 4 \pi k r^{2} \\
\frac{\partial}{\partial r}\left(\frac{g(r, \theta)}{r^{2}}\right) & \leq 0
\end{aligned}
$$

for all $\theta, \vartheta \in \mathbb{R}$ and positive $r \in \mathbb{R}$. To show (41), note that $g$ is $\theta_{k}$-periodic in the second argument

$$
g\left(r, \theta+\theta_{k}\right)=g(r, \theta)
$$

which can be verified using the identities:

$$
g_{i+n}(r, \theta)=g_{i}(r, \theta), \quad g_{i}\left(r, \theta+\theta_{k}\right)=g_{i-1}(r, \theta) .
$$

By the mean value theorem, for each $\theta, \vartheta \in \mathbb{R}$, there exists $\eta_{i} \in \mathbb{R}$ such that

$$
g_{i}(r, \theta)-g_{i}(r, \vartheta)=\frac{\partial g_{i}}{\partial \theta}\left(r, \eta_{i}\right)(\theta-\vartheta)
$$


Let

$$
s_{i}:=r \sin \left(i \theta_{k}+\eta_{i}\right), \quad c_{i}:=r \cos \left(i \theta_{k}+\eta_{i}\right) .
$$

Then, using part 5) of Assumption 2,

$$
\begin{aligned}
& \left|g_{i}(r, \theta)-g_{i}(r, \vartheta)\right| \\
= & \left|\frac{\partial g_{i}}{\partial \theta}\left(r, \eta_{i}\right)(\theta-\vartheta)\right|=\left|\left(s_{i} c_{i} \psi^{\prime}\left(s_{i}\right)+c_{i} \psi\left(s_{i}\right)\right)(\theta-\vartheta)\right| \\
\leq & \left(\left|s_{i} c_{i}\right|\left|\psi^{\prime}\left(s_{i}\right)\right|+\left|c_{i}\right|\left|\psi\left(s_{i}\right)\right|\right)|\theta-\vartheta| \leq 2 r^{2}|\theta-\vartheta| .
\end{aligned}
$$

Noting the periodicity (43) and taking the summation over $i \in \mathbb{Z}_{n}$, we have

$$
|g(r, \theta)-g(r, \vartheta)| \leq \sum_{i=1}^{n} 2 r^{2} \theta_{k}=4 \pi k r^{2}
$$

proving (41).

To prove condition (40), note that

$$
|f(r, \theta)-f(r, \vartheta)| \leq 4 \pi k r^{2}
$$

holds for all $\theta, \vartheta, r \in \mathbb{R}$ such that $r>0$, which can be shown in a manner similar to (41). It then suffices to prove that $f(r, 0)=0$. Note that (44) holds when $g$ is replaced by $f$, and hence, we have

$$
\begin{aligned}
f_{i}(r, 0) & =f_{i-n}(r, 0)=-f_{n-i}(r, 0) \\
f(r, 0) & =\sum_{i=1}^{n} f_{i}(r, 0)=\sum_{i=0}^{n-1} f_{n-i}(r, 0)=\sum_{i=1}^{n} f_{n-i}(r, 0) .
\end{aligned}
$$

Therefore,

$$
f(r, 0)=\frac{1}{2} \sum_{i=1}^{n}\left[f_{i}(r, 0)+f_{n-i}(r, 0)\right]=0 .
$$

Finally, condition (42) simply follows from

$$
\frac{\partial}{\partial r}\left(\frac{g(r, \theta)}{r^{2}}\right)=\sum_{i=1}^{n} \frac{x_{i}^{2}}{r^{3}}\left(\psi^{\prime}\left(x_{i}\right)-\frac{\psi\left(x_{i}\right)}{x_{i}}\right) \leq 0
$$

with $x_{i}:=r \sin \left(i \theta_{k}+\theta\right)$ and noting part 6) of Assumption 2.

We now prove (15) using (39)-(42). Let $(r, \theta)$ be a limit cycle orbit defined by (8) and (12). Then, it satisfies (39), which can be rewritten as

$$
\begin{gathered}
\frac{\dot{r}}{r}=\gamma-\omega_{o} \rho, \quad \dot{\theta}-\omega_{o}=\rho+\omega_{o} \gamma \\
\gamma(r, \theta):=\frac{2 g(r, \theta)}{n \hbar_{o} r^{2}}-1, \quad \rho(r, \theta):=\frac{2 f(r, \theta)}{n \hbar_{o} r^{2}} .
\end{gathered}
$$

We claim that, with $\mu_{n}:=8 \pi k /\left(n \hbar_{o}\right)$ :

$$
|\gamma(r, \theta)| \leq\left(1+\left|\omega_{o}\right|\right) \mu_{n}, \quad \rho(r, \theta) \leq \mu_{n} \quad \forall t \in \mathbb{R} .
$$

The bound on $\rho$ follows directly from (40). To prove the bound on $\gamma$, let $\hat{t} \in \mathbb{R}$ be a time instant at which the radius is maximum, i.e., $r(t) \leq r(\hat{t})$ for all $t \in \mathbb{R}$, and define $\hat{r}:=r(\hat{t})$, and $\hat{\theta}:=$ $\theta(\hat{t})$. Similarly, let $\check{t}$ be a time instant at which the radius is minimum, and define $\check{r}$ and $\check{\theta}$ as before. By the monotonicity property in (42), we have

$$
\gamma(\hat{r}, \theta) \leq \gamma(r, \theta) \leq \gamma(\check{r}, \theta)
$$

for all $t$. Let $(\bar{t}, \bar{r}, \bar{\theta})$ be either $(\check{t}, \check{r}, \check{\theta})$ or $(\hat{t}, \hat{r}, \hat{\theta})$. Then, noting that $\dot{r}(\bar{t})=0$, we have $\gamma(\bar{r}, \bar{\theta})=\omega_{o} \rho(\bar{r}, \bar{\theta})$. Using (40), (41), and this relation:

$$
|\gamma(\bar{r}, \theta)| \leq|\gamma(\bar{r}, \bar{\theta})|+|\gamma(\bar{r}, \theta)-\gamma(\bar{r}, \bar{\theta})| \leq\left(1+\left|\omega_{o}\right|\right) \mu_{n} .
$$

Hence, we have the bound on $\gamma$ as in (46). As a result

$$
\begin{aligned}
& \left|\begin{array}{l}
\dot{r} \\
r
\end{array}\right| \leq|\gamma|+\left|\omega_{o} \rho\right| \leq\left(1+2\left|\omega_{o}\right|\right) \mu_{n} \\
& \left|\dot{\theta}-\omega_{o}\right|=|\rho|+\left|\omega_{o} \gamma\right| \leq\left(1+\left|\omega_{o}\right|+\omega_{o}^{2}\right) \mu_{n}
\end{aligned}
$$

hold for all $t$. Now, consider the limit where $n$ goes to infinity. If $r$ approaches $\alpha_{o}$, then the results follow by noting that $\mu_{n}$ approaches zero. Hence, it remains to show $r \rightarrow \alpha_{o}$. First, we note that $\kappa(r)$ is a strictly decreasing function due to part 6) of Assumption 2 . Thus, $\alpha_{o}$ is uniquely determined by $\kappa\left(\alpha_{o}\right)=\hbar_{o}$. Therefore, $r$ approaches $\alpha_{o}$ if and only if $\kappa(r)$ approaches $\hbar_{o}$. By definition of the describing function, we have

$$
\lim _{n \rightarrow \infty} \frac{2 g(r, \theta)}{n r^{2}}=\kappa(r)
$$

for all $r>0$ and $\theta$. On the other hand, when $(r, \theta)$ is an orbit of (8)

$$
\lim _{n \rightarrow \infty} \frac{2 g(r, \theta)}{n r^{2}}=\lim _{n \rightarrow \infty}(\gamma(r, \theta)+1) \hbar_{o}=\hbar_{o}
$$

where we used (46). This completes the proof.

\section{ACKNOWLEDGMENT}

The authors gratefully acknowledge helpful discussions with Prof. A. Megretski and Prof. J. J. Slotine at Massachusetts Institute of Technology (MIT).

\section{REFERENCES}

[1] A. H. Cohen, G. B. Ermentrout, T. Kiemel, N. Kopell, K. A. Sigvardt, and T. L. Williams, "Modelling of intersegmental coordination in the lamprey central pattern generator for locomotion," Trends Neurosci., vol. 15, no. 11, pp. 434-438, 1992.

[2] G. B. Ermentrout and C. C. Chow, "Modeling neural oscillations," Physiol. Behav., vol. 77, pp. 629-633, 2002.

[3] W. O. Friesen, G. D. Block, and C. G. Hocker, "Formal approaches to understanding biological oscillators," Ann.Rev. Physiol., vol. 55, pp. 661$681,1993$.

[4] G. N. Orlovsky, T. G. Deliagina, and S. Grillner, Neuronal Control of Locomotion: From Mollusc to Man. London, U.K.: Oxford Univ. Press, 1999.

[5] F. K. Skinner and B. Mulloney, "Intersegmental coordination in invertebrates and vertebrates," Curr. Opin. Neurobiol., vol. 8, pp. 725-732, 1998.

[6] J. Cang and W. O. Friesen, "Model for intersegmental coordination of leech swimming: Central sensory mechanisms," J. Neurophysiol., vol. 87, pp. $2760-2769,2002$.

[7] A. H. Cohen and D. L. Boothe, "Sensory motor interactions during locomotion: Principles derived from biological systems," Auton. Robots, vol. 7, no. 3, pp. 239-245, 1999.

[8] T. Iwasaki and M. Zheng, "Sensory feedback mechanism underlying entrainment of central pattern generator to mechanical resonance," Biol. Cybern., vol. 94, no. 4, pp. 245-261, 2006.

[9] Y. Fukuoka, H. Kimura, and A. H. Cohen, "Adaptive dynamic walking of a quadruped robot on irregular terrain based on biological concepts," Int. J. Robot. Res., vol. 22, no. 3/4, pp. 187-202, 2003.

[10] M. M. Williamson, "Neural control of rhythmic arm movements," Neural Netw., vol. 11, pp. 1379-1394, 1998. 
[11] G. Taga, "Self-organized control of bipedal locomotion by neural oscillators in unpredictable environment," Biol. Cybern., vol. 65, pp. 147-159, 1991.

[12] M. A. Lewis and G. A. Bekey, "Gait adaptation in a quadruped robot," Auton. Robots, vol. 12, no. 3, pp. 301-312, 2002.

[13] E. R. Westervelt, J. W. Grizzle, and D. E. Koditschek, "Hybrid zero dynamics of planar biped walkers," IEEE Trans. Autom. Control, vol. 48, no. 1, pp. 42-56, Jan. 2003.

[14] R. W. Brockett, "Pattern generation and the control of nonlinear systems," IEEE Trans. Autom. Control, vol. 48, no. 10, pp. 1699-1711, Oct. 2003.

[15] U. T. Jonsson, C. Y. Kao, and A. Megretski, "Robustness of periodic trajectories," IEEE Trans. Autom. Control, vol. 47, no. 11, pp. 18421856, Nov. 2002.

[16] P. Arena, L. Fortuna, and M. Frasca, "Multi-template approach to realize central pattern generators for artificial locomotion control," Int. J. Circuit Theory Appl., vol. 30, pp. 441-458, 2002.

[17] W. Wang and J. J. E. Slotine, "On partial contraction analysis for coupled nonlinear oscillators,” Biol. Cybern., vol. 92, no. 1, pp. 38-53, 2005.

[18] Q. C. Pham and J. J. Slotine. (2005). Stable concurrent synchronization in dynamic system networks. Quant. Biol. [Online]. Available: http://arxiv. org/abs/q-bio.NC/0510051

[19] G. B. Stan and R. Sepulchre, "Analysis of interconnected oscillators by dissipativity theory," IEEE Trans. Autom. Control, vol. 52, no. 2, pp. 256270, Feb. 2007.

[20] J. A. Marshall, M. E. Broucke, and B. A. Francis, "Formations of vehicles in cyclic pursuit," IEEE Trans. Autom. Control, vol. 49, no. 11, pp. 19631974, Nov. 2004.

[21] P. J. Davis, Circulant Matrices, 2nd ed. New York: Chelsea, 1994.

[22] I. S. Gradshteyn and I. M. Ryzhik, Tables of Integrals, Series, and Products, 6th ed. New York: Academic, 2000.

[23] E. W. Weisstein. (2005). Circulant determinant [Online]. Available: MathWorld-Wolfram Web Resource, http://mathworld.wolfram.com/ CirculantDeterminant.html

[24] T. Iwasaki, "Analysis and synthesis of central pattern generators via multivariable harmonic balance," in Proc. Amer. Control Conf., Minneapolis, MN, Jun. 14-16, 2006, pp. 4106-4111.

[25] H. K. Khalil, Nonlinear Systems. Englewood Cliffs, NJ: Prentice-Hall, 1996.

[26] M. Saito, M. Fukaya, and T. Iwasaki, "Serpentine locomotion with robotic snake," IEEE Control Syst. Mag., vol. 22, no. 1, pp. 64-81, Feb. 2002

[27] S. Hirose, Biologically Inspired Robots: Snake-Like Locomotors and Manipulators. London, U.K.: Oxford Univ. Press, 1993.

[28] R. Genesio and A. Tesi, "Harmonic balance methods for the analysis of chaotic dynamics in nonlinear systems," Automatica, vol. 28, no. 3, pp. 531-548, 1992.

[29] N. Kopell, "Toward a theory of modeling central pattern generators," in Neural Control of Rhythmic Movements in Vertebrates, A. H. Cohen, S. Rossignol, and S. Grillner, Eds. New York: Wiley/Interscience, 1987, pp. 369-413.

[30] G. Hicks and K. Ito, "A method for determination of optimal gaits with application to a snake-like serial-link structure," IEEE Trans. Autom Control, vol. 50, no. 9, pp. 1291-1306, Sep. 2005.
[31] M. Zheng, W. O. Friesen, and T. Iwasaki, "Systems-level modeling of neuronal circuits for leech swimming," J. Comput. Neurosci., vol. 22 , no. 1, pp. 21-38, 2007 (DOI:10.1007/s10827-006-9648-7).

[32] S. Inagaki, H. Yuasa, and T. Arai, "CPG model for autonomous decentralized multi-legged robot system-Generation and transition of oscillation patterns and dynamics of oscillators," Robot. Auton. Syst., vol. 44, no. 3/4, pp. 171-179, 2003.

[33] Z. Yang and F. M. G. Franca, "A generalized locomotion CPG architecture based on oscillatory building blocks," Biol. Cybern., vol. 89, no. 1, pp. 34 42, 2003.

[34] G. Hardy, J. Littlewood, and G. Polya, Inequalities. Cambridge, U.K.: Cambridge Univ. Press, 1988.

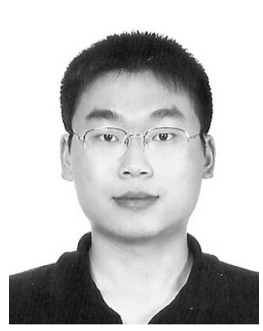

Zhiyong Chen (S'02-M'05) received the B.S. degree in automatic control from the Department of Automation, University of Science and Technology of China, Hefei, China, in 2000, and the M. Phil. and Ph.D. degrees in mechanical and automation engineering from the Chinese University of Hong Kong, Hong Kong, SAR, in 2002 and 2005, respectively.

During 2005-2006, he was a Research Associate at the University of Virginia, Charlottesville. He is currently a Lecturer at the University of Newcastle, Newcastle, N.S.W., Australia. His current research interests include biological and nonlinear systems and control.

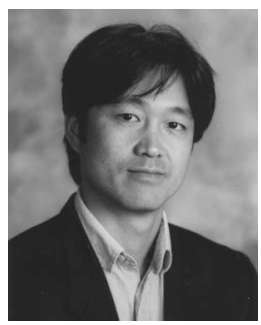

Tetsuya Iwasaki (S'89-M'90-SM'01) received the B.E. and M.E. degrees in electrical and electronic engineering from Tokyo Institute of Technology, Tokyo, Japan, in 1987 and 1990, respectively, and the $\mathrm{Ph} . \mathrm{D}$. degree in dynamics and control from the School of Aeronautics and Astronautics, Purdue University, West Lafayette, IN, in 1993.

He was a faculty member at Tokyo Institute of Technology, for several years. In 2000, he joined the University of Virginia, Charlottesville, where he is currently a Professor. He is currently a member of the editorial board of the Systems and Control Letters and the International Journal of Robust and Nonlinear Control. He was an Associate Editor of the IFAC Automatica. His current research interests include robust and optimal control, and modeling and analysis of neuronal control systems for animal locomotion.

Prof. Iwasaki is the recipient of the 2002 Pioneer Prize from the Society of Instrument and Control Engineers, the 2003 National Science Foundation (NSF) CAREER Award, and the 2006 George S. Axelby Outstanding Paper Award. He was an Associate Editor of the IEEE TRANSACTIONS ON AUTOMATIC CONTROL. 